\title{
MULTIGRADED CASTELNUOVO-MUMFORD REGULARITY
}

\author{
DIANE MACLAGAN AND GREGORY G. SMITH
}

\begin{abstract}
We develop a multigraded variant of Castelnuovo-Mumford regularity. Motivated by toric geometry, we work with modules over a polynomial ring graded by a finitely generated abelian group. As in the standard graded case, our definition of multigraded regularity involves the vanishing of graded components of local cohomology. We establish the key properties of regularity: its connection with the minimal generators of a module and its behavior in exact sequences. For an ideal sheaf on a simplicial toric variety $X$, we prove that its multigraded regularity bounds the equations that cut out the associated subvariety. We also provide a criterion for testing if an ample line bundle on $X$ gives a projectively normal embedding.
\end{abstract}

\section{INTRODUCTION}

Castelnuovo-Mumford regularity is a fundamental invariant in commutative algebra and algebraic geometry. Intuitively, it measures the complexity of a module or sheaf. The regularity of a module approximates the largest degree of the minimal generators and the regularity of a sheaf estimates the smallest twist for which the sheaf is generated by its global sections. Although the precise definition may seem rather technical, its value in bounding the degree of syzygies GLP EL and constructing Hilbert schemes Got [K] has established that regularity is an indispensable tool in both fields.

The goal of this paper is to develop a multigraded variant of Castelnuovo-Mumford regularity. We work with modules over a polynomial ring graded by a finitely generated abelian group. Imitating [EG], our definition of regularity involves the vanishing of certain graded components of local cohomology. We establish the key properties of regularity: its connection with the minimal generators of a module and its behavior in short exact sequences. As a consequence, we are able relate the regularity of a module to chain complexes associated with the module.

Our primary motivation for studying regularity over multigraded polynomial rings comes from toric geometry. For a simplicial toric variety $X$, the homogeneous coordinate ring, introduced in [Co2], is a polynomial ring $S$ graded by the divisor class group $G$ of $X$. The dictionary linking the geometry of $X$ with the theory of $G$-graded $S$-modules leads to geometric interpretations and applications for multigraded regularity. We prove that the regularity of an ideal sheaf bounds the multidegrees of the equations that cut out the corresponding subvariety. Multigraded regularity also supplies a criterion for testing if an ample line bundle on $X$ determines a projectively normal embedding.

Multigraded regularity consolidates a range of existing ideas. In the standard graded case, it reduces to Castelnuovo-Mumford regularity. If $S$ has a nonstandard $\mathbb{Z}$-grading, then our definition is the version of regularity introduced in Ben] for studying group cohomology. When $S$ is the homogeneous coordinate ring of a product of projective spaces, multigraded regularity is the weak form of bigraded regularity defined in [HW]. 
Our description for the multigraded regularity of fat points (Proposition 6.7) is also connected with the results in [HV]. On the other hand, the versions of regularity developed for Grassmannians in [Chi] and abelian varieties in [PP] do not fall under the umbrella of multigraded regularity.

For ease of exposition we state our theorems in the case where $S$ is the homogeneous coordinate ring of a smooth projective toric variety $X$. Let $B$ be the irrelevant ideal of $X$. We denote by $\mathbb{N} \mathcal{C}$ the semigroup generated by a finite subset $\mathcal{C}=\left\{\boldsymbol{c}_{1}, \ldots, \boldsymbol{c}_{\ell}\right\}$ of $G \cong \operatorname{Pic}(X)$. In the introduction, we restrict to the special case that $\mathcal{C}$ is the minimal generating set of the semigroup of nef line bundles on $X$. For example, if $X=\mathbb{P}^{d}$ then $G \cong \mathbb{Z}, S$ has the standard grading defined by $\operatorname{deg}\left(x_{i}\right)=\mathbf{1}$ for $1 \leq i \leq n$, $B=\left\langle x_{1}, \ldots, x_{n}\right\rangle$, and $\mathcal{e}=\{\mathbf{1}\}$.

The main point of this paper is to introduce the following definition, which generalizes Castelnuovo-Mumford regularity.

Definition 1.1. For $\boldsymbol{m} \in G$, we say that a $G$-graded $S$-module $M$ is $\boldsymbol{m}$-regular (with respect to $\mathcal{C})$ if the following conditions are satisfied:

1. $H_{B}^{i}(M)_{\boldsymbol{p}}=0$ for all $i \geq 1$ and all $\boldsymbol{p} \in \bigcup\left(\boldsymbol{m}-\lambda_{1} \boldsymbol{c}_{1}-\cdots-\lambda_{\ell} \boldsymbol{c}_{\ell}+\mathbb{N} \mathcal{C}\right)$ where the union is over all $\lambda_{1}, \ldots, \lambda_{\ell} \in \mathbb{N}$ such that $\lambda_{1}+\cdots+\lambda_{\ell}=i-1$;

2. $H_{B}^{0}(M)_{\boldsymbol{p}}=0$ for all $\boldsymbol{p} \in \bigcup_{1 \leq j \leq \ell}\left(\boldsymbol{m}+\boldsymbol{c}_{j}+\mathbb{N} \boldsymbol{C}\right)$.

We set $\operatorname{reg}(M):=\{\boldsymbol{p} \in G: M$ is $\boldsymbol{p}$-regular $\}$.

In contrast with the usual notation, $\operatorname{reg}(M)$ is a set rather than a single group element. Traditionally, $G=\mathbb{Z}$ and the regularity of $M$ refers to the smallest $\boldsymbol{m} \in G$ such that $M$ is $\boldsymbol{m}$-regular. When $S$ has a multigrading, the group $G$ is not equipped with a natural ordering so one cannot choose a smallest degree $\boldsymbol{m}$. More significantly, the set $\operatorname{reg}(M)$ may not even be determined by a single element; see Example 1.2. From this vantage point, bounding the regularity of a module $M$ is equivalent to giving a subset of $\operatorname{reg}(M)$.

Example 1.2. When $X$ is the Hirzebruch surface $\mathbb{F}_{2}$, the homogeneous coordinate ring $S=\mathbb{k}\left[x_{1}, x_{2}, x_{3}, x_{4}\right]$ has the $\mathbb{Z}^{2}$-grading defined by $\operatorname{deg}\left(x_{1}\right)=\left[\begin{array}{l}1 \\ 0\end{array}\right], \operatorname{deg}\left(x_{2}\right)=\left[\begin{array}{c}-2 \\ 0\end{array}\right]$, $\operatorname{deg}\left(x_{3}\right)=\left[\begin{array}{l}1 \\ 0\end{array}\right]$ and $\operatorname{deg}\left(x_{4}\right)=\left[\begin{array}{l}0 \\ 1\end{array}\right]$ and the irrelevant ideal $B$ is $\left\langle x_{1} x_{2}, x_{2} x_{3}, x_{3} x_{4}, x_{1} x_{4}\right\rangle$. This grading identifies $\operatorname{Pic}(X)$ with $\mathbb{Z}^{2}$. It follows that the semigroup of nef line bundles is generated by the set $\mathcal{C}=\left\{\left[\begin{array}{l}1 \\ 0\end{array}\right],\left[\begin{array}{l}0 \\ 1\end{array}\right]\right\}$. A topological interpretation for $H_{B}^{i}(S)$ (see Section 3) shows that $\operatorname{reg}(S)=\left(\left[\begin{array}{l}1 \\ 0\end{array}\right]+\mathbb{N}^{2}\right) \cup\left(\left[\begin{array}{l}0 \\ 1\end{array}\right]+\mathbb{N}^{2}\right)$. A graphical representation of $\operatorname{reg}(S)$ appears in Figure 5. Observe that $\operatorname{reg}(S)$ is not determined by a single element of $G$ and $\left[\begin{array}{l}0 \\ 0\end{array}\right] \notin \operatorname{reg}(S)$.

The following key result shows that the regularity of a module is a cohomological approximation for the degrees of its minimal generators.

Theorem 1.3. Let $M$ be a finitely generated $G$-graded $S$-module. If the module $M$ is $\boldsymbol{m}$-regular, then the degrees of the minimal generators of $M$ do not belong to the set $\operatorname{reg}(M)+\left(\bigcup_{1 \leq j \leq \ell}\left(\boldsymbol{c}_{j}+\mathbb{N} \boldsymbol{e}\right)\right)$.

To emphasize the similarities between the multigraded form of regularity and the original definition in $\S 14$ of $\mathrm{Mum}$, we give the geometric translation of this result below. For $\boldsymbol{p} \in G, \mathscr{O}_{X}(\boldsymbol{p})$ is the associated line bundle on $X$. Set $\mathscr{F}(\boldsymbol{p}):=\mathscr{F} \otimes \mathscr{O}_{X}(\boldsymbol{p})$.

Theorem 1.4. Let $\mathscr{F}$ be a coherent $\mathscr{O}_{X}$-module. If $\mathscr{F}$ is $\boldsymbol{m}$-regular then for every $\boldsymbol{p} \in \boldsymbol{m}+\mathbb{N} \mathcal{C}$ we have the following: 
1. $\mathscr{F}$ is $\boldsymbol{p}$-regular;

2. the natural map $H^{0}(X, \mathscr{F}(\boldsymbol{p})) \otimes H^{0}\left(X, \mathscr{O}_{X}(\boldsymbol{q})\right) \rightarrow H^{0}(X, \mathscr{F}(\boldsymbol{p}+\boldsymbol{q}))$ is surjective for all $\boldsymbol{q} \in \mathbb{N} \mathcal{C}$;

3. $\mathscr{F}(\boldsymbol{p})$ is generated by its global sections.

We highlight two consequences of this theorem. Firstly, if $\mathscr{I}$ is an ideal sheaf on $X$ and $\boldsymbol{m} \in \operatorname{reg}(\mathscr{I})$ then the subscheme of $X$ defined by $\mathscr{I}$ is cut out by equations of degree $\boldsymbol{m}$. Secondly, if $\mathscr{O}_{X}(\boldsymbol{p})$ is an ample line bundle and $\boldsymbol{p} \in \operatorname{reg}\left(\mathscr{O}_{X}\right)$ then the complete linear series associated to $\mathscr{O}_{X}(\boldsymbol{p})$ gives a projectively normal embedding of $X$. In particular, if $\mathbf{0} \in \operatorname{reg}\left(\mathscr{O}_{X}\right)$ then every ample line bundle on $X$ gives a projectively normal embedding.

The next result illustrates a second key feature of regularity, namely its behavior in exact sequences. When $S$ has the standard grading, the following are equivalent: the module $M$ is $\boldsymbol{m}$-regular, the degrees of the $i$ th syzgies are at most $\boldsymbol{m}+i$, and the truncated module $\left.M\right|_{\geq \boldsymbol{m}}$ has a linear resolution. We generalize these properties in the following way.

Theorem 1.5. Let $d$ be the number of variable in polynomial ring $S$ minus the rank of group $G$ and let $M$ be a finitely generated $G$-graded $S$-module.

1. If $\cdots \longrightarrow E_{3} \longrightarrow E_{2} \longrightarrow E_{1} \longrightarrow E_{0} \stackrel{\partial_{0}}{\longrightarrow} M \longrightarrow 0$ is a chain complex of finitely generated $G$-graded $S$-modules with $B$-torsion homology and $\partial_{0}$ is surjective then

$$
\bigcup_{\phi:[d+1] \rightarrow[\ell]}\left(\bigcap_{1 \leq i \leq d+1}\left(-\boldsymbol{c}_{\phi(1)}-\cdots-\boldsymbol{c}_{\phi(i)}+\operatorname{reg}\left(E_{i}\right)\right)\right) \subseteq \operatorname{reg}(M) .
$$

2. If $\boldsymbol{c} \in \operatorname{reg}(S) \cap\left(\bigcap_{1 \leq j \leq \ell}\left(\boldsymbol{c}_{j}+\mathbb{N} \boldsymbol{C}\right)\right)$ and $\boldsymbol{m} \in \operatorname{reg}(M)$ then there exists a chain complex $\quad \cdots \longrightarrow E_{3} \longrightarrow E_{2} \longrightarrow E_{1} \longrightarrow E_{0} \longrightarrow M \longrightarrow 0$ with B-torsion homology and $E_{i}=\bigoplus S(-\boldsymbol{m}-i \boldsymbol{c})$.

If $S$ has the standard grading, then the inclusion in Part 1 is an equality when the $E_{i}$ form a minimal free resolution of $M$, and the chain complex in Part 2 is the minimal free resolution of $\left.M\right|_{\geq \boldsymbol{m}}$. Since $B$-torsion modules correspond to the zero sheaf on the corresponding toric variety, there is also a geometric version of this theorem involving regularity and resolutions of a sheaf.

The techniques used to establish Theorems 1.3, 1.4 and 1.5 also apply to a larger class of multigraded polynomial rings. We develop these methods for pairs $(S, B)$ where $S$ is a polynomial ring graded by a finitely generated abelian group $G$, and $B$ is a monomial ideal that encodes a certain combinatorial structure of the grading. This class of rings includes the homogeneous coordinate rings of simplicial semi-projective toric varieties. We also establish these results for other choices of the set $\mathcal{C}$.

This paper is organized as follows. In the next section, we discuss the basic definitions and examples of multigraded polynomial rings. In Section 3, we establish some vanishing theorems for local cohomology modules. These results are based on a topological description for the multigraded Hilbert series of $H_{B}^{i}(S)$. The definition of multigraded regularity is presented in Section 4 . We also prove that in certain cases the definition of regularity is equivalent to an apparently weaker vanishing condition. Section 5 connects the multigraded regularity of a module with the degrees of its minimal generators. In 
Section 6, we reinterpret regularity of $S$-module in terms of coherent sheaves on a simplicial toric variety and study some geometric applications. Finally, Section 7 examines the relationship between chain complexes associated to a module or sheaf and regularity.

Acknowledgements. We thank Dave Benson for conversations which expanded our notion of regularity. We are also grateful to Kristina Crona for conversations about multigradings early in this project. The computer software package Macaulay 2 [M2] was indispensable for computing examples. Both authors were partially supported by the Mathematical Sciences Research Institute in Berkeley, CA.

\section{Multigraded Polynomial Rings}

In this section, we develop the foundations of multigraded polynomial rings. Let $\mathbb{k}$ be a field and let $G$ be a finitely generated abelian group. Throughout this paper, we work with a pair $(S, B)$ where $S:=\mathbb{k}\left[x_{1}, \ldots, x_{n}\right]$ is a $G$-graded polynomial ring and $B$ is a monomial ideal in $S$. For a positive integer $m$, we write $[m]$ for the set $\{1, \ldots, m\}$. The convex cone generated by (or positive hull of) the vectors $\left\{\boldsymbol{v}_{1}, \ldots, \boldsymbol{v}_{m}\right\}$ is the set $\operatorname{pos}\left(\boldsymbol{v}_{1}, \ldots, \boldsymbol{v}_{m}\right):=\left\{\lambda_{1} \boldsymbol{v}_{1}+\cdots+\lambda_{m} \boldsymbol{v}_{m}: \lambda_{1}, \ldots, \lambda_{m} \in \mathbb{R}_{\geq 0}\right\}$.

A $G$-grading of the polynomial ring $S=\mathbb{k}\left[x_{1}, \ldots, x_{n}\right]$ corresponds to a semigroup homomorphism $\mathbb{N}^{n} \longrightarrow G$. We say a monomial $\boldsymbol{x}^{\boldsymbol{u}}$ has degree $\boldsymbol{p}$ if $\boldsymbol{u} \longmapsto \boldsymbol{p} \in G$. This map induces a decomposition $S=\bigoplus_{\boldsymbol{p} \in G} S_{\boldsymbol{p}}$ satisfying $S_{\boldsymbol{p}} \cdot S_{\boldsymbol{q}} \subseteq S_{\boldsymbol{p}+\boldsymbol{q}}$ where $S_{\boldsymbol{p}}$ is the $\mathbb{k}$-span of all $\boldsymbol{x}^{\boldsymbol{u}}$ of degree $\boldsymbol{p}$. The $G$-grading is determined by the set $\mathcal{A}:=\left\{\boldsymbol{a}_{1}, \ldots, \boldsymbol{a}_{n}\right\}$ where $\boldsymbol{a}_{i}:=\operatorname{deg}\left(x_{i}\right) \in G$ for $1 \leq i \leq n$. We write $\mathbb{N} \mathcal{A}$ for the subsemigroup of $G$ generated by $\mathcal{A}$. Let $r$ be the rank of $G$ and identify $\mathbb{R} \otimes_{\mathbb{Z}} G$ with $\mathbb{R}^{r}$. If $\overline{\boldsymbol{a}}_{i}$ denotes the image of $\boldsymbol{a}_{i}$ in $\mathbb{R} \otimes_{\mathbb{Z}} G=\mathbb{R}^{r}$, then the set $\overline{\mathcal{A}}:=\left\{\overline{\boldsymbol{a}}_{1}, \ldots, \overline{\boldsymbol{a}}_{n}\right\}$ is an integral vector configuration in $\mathbb{R}^{r}$. The monomial ideal $B$ corresponds to a chamber (maximal cell) $\Gamma \subset \mathbb{R}^{r}$ in the chamber complex of the vector configuration $\overline{\mathcal{A}}$. The chamber complex is the coarsest fan with support $\operatorname{pos}(\overline{\mathcal{A}})$ that refines all the triangulations of $\overline{\mathcal{A}}$; see BGS. We encode the choice of a chamber $\Gamma$ in the monomial ideal

$$
B:=\left\langle\prod_{i \in \sigma} x_{i}: \sigma \subseteq[n] \text { with } \Gamma \subseteq \operatorname{pos}\left(\overline{\boldsymbol{a}}_{i}: i \in \sigma\right)\right\rangle .
$$

Alternatively, the monomial ideal $B$ can be described by a regular triangulation (Definition 5.3 in Zie) of the dual vector configuration. If $d:=n-r$, then the dual vector configuration is a set of vectors $\overline{\mathcal{B}}:=\left\{\overline{\boldsymbol{b}}_{1}, \ldots, \overline{\boldsymbol{b}}_{n}\right\}$ in $\mathbb{R}^{d}$ such that

$$
0 \longrightarrow \mathbb{R}^{d \stackrel{\left[\overline{\boldsymbol{b}}_{1} \cdots \overline{\boldsymbol{b}}_{n}\right]^{\top}}{\longrightarrow}} \mathbb{R}^{n} \stackrel{\left[\overline{\boldsymbol{a}}_{1} \cdots \overline{\boldsymbol{a}}_{n}\right]}{\longrightarrow} \mathbb{R}^{r} \longrightarrow 0
$$

is a short exact sequence. The set $\overline{\mathcal{B}}$ is uniquely determined up to a linear change of coordinates on $\mathbb{R}^{d}$; see $\S 6.4$ in [Zie. We identify a triangulation of $\overline{\mathcal{B}}$ with a simplicial complex $\Delta$. Gale duality, specifically Theorem 3.1 in BGS, implies that the chamber $\Gamma \subset \mathbb{R}^{r}$ corresponds to a regular triangulation $\Delta$ of $\overline{\mathcal{B}}$. For $\sigma \subseteq[n]$, let $\widehat{\sigma}$ denote the complement of $\sigma$ in $[n]$. From this standpoint, we have $B=\left\langle\prod_{i \in \widehat{\sigma}} x_{i}: \sigma \in \Delta\right\rangle$.

The simplicial complex $\Delta$ (or equivalently the chamber $\Gamma$ ) also gives rise to two important subsemigroups of $\mathbb{N} \mathcal{A}$. The first subsemigroup $\mathcal{K}$ is the intersection $\bigcap_{\sigma \in \Delta} \mathbb{N} \mathcal{A}_{\widehat{\sigma}}$ where $\mathcal{A}_{\widehat{\sigma}}:=\left\{\boldsymbol{a}_{i}: i \in \widehat{\sigma}\right\}$. Let $\mathbb{Z} \mathcal{A}$ be the subgroup of $G$ generated by $\mathcal{A}$. The second subsemigroup $\mathcal{K}^{\text {sat }}$ is the saturation (or normalization) of $\mathcal{K}$ in $\mathbb{Z} \mathcal{A}$. In other words, $\mathcal{K}^{\text {sat }}$ is the set of all $\boldsymbol{p} \in \mathbb{N} \mathcal{A}$ such that the image $\overline{\boldsymbol{p}} \in \mathbb{R}^{r}$ lies in the chamber $\Gamma$. The 
interior of $\mathcal{K}^{\text {sat }}$, denoted int $\mathcal{K}^{\text {sat }}$, consists of all $\boldsymbol{p} \in \mathcal{K}^{\text {sat }}$ such that $\overline{\boldsymbol{p}}$ lies in the interior of $\Gamma$.

Example 2.1. Let $G=\mathbb{Z}$ and assume that $\boldsymbol{a}_{i}>0$ for all $i$. Since $G$ is torsion-free, we may identify $\boldsymbol{a}_{i}$ with $\overline{\boldsymbol{a}}_{i}$. The polynomial $\operatorname{ring} S=\mathbb{k}\left[x_{1}, \ldots, x_{n}\right]$ has the $\mathbb{Z}$-grading induced by $\operatorname{deg}\left(x_{i}\right)=\boldsymbol{a}_{i}$ for $1 \leq i \leq n$. The chamber complex of $\mathcal{A}$ has a unique maximal cell $\Gamma=\mathbb{R}_{\geq 0}$ and the corresponding ideal is $B=\left\langle x_{1}, \ldots, x_{n}\right\rangle$. The dual vector configuration $\overline{\mathcal{B}}$ is given by the columns of the matrix

$$
\left[\begin{array}{ccccc}
\boldsymbol{a}_{n} & 0 & \cdots & 0 & -\boldsymbol{a}_{1} \\
0 & \boldsymbol{a}_{n} & \cdots & 0 & -\boldsymbol{a}_{2} \\
\vdots & \vdots & \ddots & \vdots & \vdots \\
0 & 0 & \cdots & \boldsymbol{a}_{n} & -\boldsymbol{a}_{n-1}
\end{array}\right] .
$$

The semigroup $\mathcal{K} \subset \mathbb{Z}$ consists of all nonnegative multiples of $\operatorname{lcm}(\mathcal{A})$ and $\mathcal{K}^{\text {sat }}=\mathbb{N}$. It follows that $\mathcal{K}^{\text {sat }}=\mathcal{K}$ if and only if $\boldsymbol{a}_{i}=1$ for all $1 \leq i \leq n$. When $\boldsymbol{a}_{i}=1$ for all $i$, $S$ is standard graded polynomial ring and $B$ is the unique graded maximal ideal.

Example 2.2. Fix $t \in \mathbb{N}$. Suppose that $G=\mathbb{Z}^{2}$ and let $\mathcal{A}$ correspond to the columns of the matrix

$$
\left[\begin{array}{cccc}
1 & -t & 1 & 0 \\
0 & 1 & 0 & 1
\end{array}\right] \text {. }
$$

Again, we identify $\mathcal{A}$ with $\overline{\mathcal{A}}$. The polynomial ring $S=\mathbb{k}\left[x_{1}, x_{2}, x_{3}, x_{4}\right]$ has the $\mathbb{Z}^{2}$-grading defined by $\operatorname{deg}\left(x_{1}\right)=\left[\begin{array}{l}1 \\ 0\end{array}\right], \operatorname{deg}\left(x_{2}\right)=\left[\begin{array}{c}-t \\ 0\end{array}\right], \operatorname{deg}\left(x_{3}\right)=\left[\begin{array}{l}1 \\ 0\end{array}\right]$ and $\operatorname{deg}\left(x_{4}\right)=\left[\begin{array}{l}0 \\ 1\end{array}\right]$. There are two possible choices for the chamber $\Gamma: \mathbb{R}_{\geq 0}^{2}=\operatorname{pos}\left(\left[\begin{array}{l}1 \\ 0\end{array}\right],\left[\begin{array}{l}0 \\ 1\end{array}\right]\right)$ or pos $\left(\left[\begin{array}{c}-t \\ 1\end{array}\right],\left[\begin{array}{l}0 \\ 1\end{array}\right]\right)$ and the ideal $B$ equals $\left\langle x_{1} x_{2}, x_{2} x_{3}, x_{3} x_{4}, x_{1} x_{4}\right\rangle$ or $\left\langle x_{1} x_{2}, x_{2} x_{3}, x_{2} x_{4}\right\rangle$ respectively. The dual vector configuration is given by the columns of the matrix

$$
\left[\begin{array}{cccc}
1 & 0 & -1 & 0 \\
0 & 1 & t & -1
\end{array}\right] \text {. }
$$

Figure 1 illustrates the associated vector configurations when $t=2$. Regardless of the choice of $\Gamma$, both $\mathcal{K}^{\text {sat }}$ and $\mathcal{K}$ equal $\Gamma \cap \mathbb{Z}^{2}$.
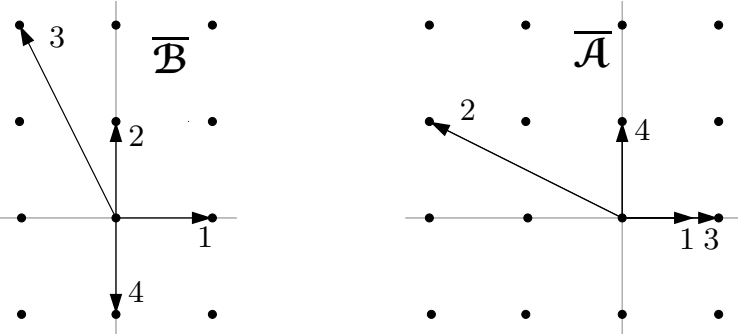

FiguRE 1. The vector configurations for Example 2.2

Example 2.3. If $G=\mathbb{Z}^{2} \oplus(\mathbb{Z} / 2 \mathbb{Z})^{2}$ and $\mathcal{A}$ corresponds to the columns of the matrix

$$
\left[\begin{array}{lllll}
1 & 0 & 1 & 0 & 1 \\
0 & 1 & 0 & 1 & 1 \\
1 & 0 & 1 & 0 & 0 \\
1 & 1 & 0 & 0 & 0
\end{array}\right]
$$


where the entries in bottom two rows are elements of $\mathbb{Z} / 2 \mathbb{Z}$, then $S=\mathbb{k}\left[x_{1}, x_{2}, x_{3}, x_{4}, x_{5}\right]$ is graded by a group with torsion. The vector configuration $\overline{\mathcal{A}}$ is given by the columns of

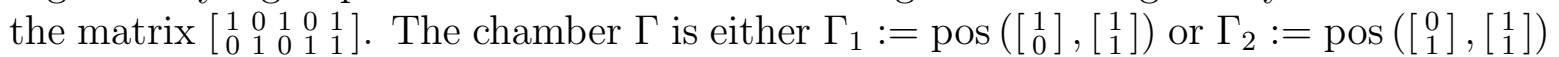
and the ideal $B$ equals either $\left\langle x_{1} x_{2}, x_{1} x_{4}, x_{1} x_{5}, x_{2} x_{3}, x_{3} x_{4}, x_{3} x_{5}\right\rangle$ in the first case or $\left\langle x_{1} x_{2}, x_{2} x_{3}, x_{2} x_{5}, x_{1} x_{4}, x_{3} x_{4}, x_{4} x_{5}\right\rangle$ in the second. The dual vector configuration $\overline{\mathcal{B}}$ is given by the columns of the matrix

$$
\left[\begin{array}{ccccc}
1 & 1 & -1 & -1 & 0 \\
1 & -1 & -1 & 1 & 0 \\
1 & 1 & 1 & 1 & -2
\end{array}\right]
$$

The two triangulations are illustrated in Figure 2. If $\Gamma_{1}$ is the chosen chamber, then

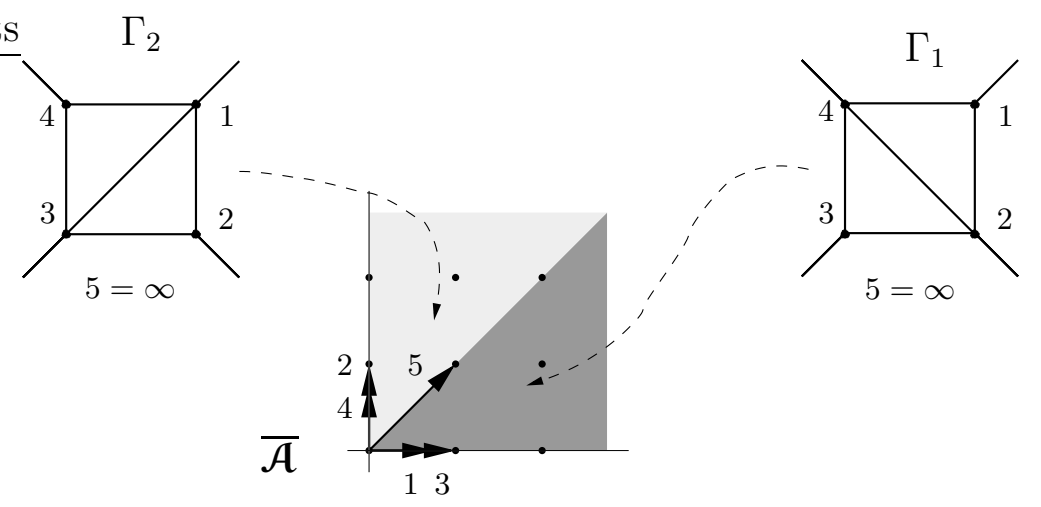

Figure 2. The two triangulations and chamber complex for Example 2.3

$$
\mathcal{K}=\mathbb{N}\left\{\left[\begin{array}{l}
2 \\
0 \\
0 \\
0
\end{array}\right],\left[\begin{array}{l}
2 \\
2 \\
0 \\
0
\end{array}\right]\right\} \quad \text { and } \quad \mathcal{K}^{\text {sat }}=\mathbb{N}\left\{\left[\begin{array}{l}
1 \\
0 \\
0 \\
0
\end{array}\right],\left[\begin{array}{l}
1 \\
1 \\
0 \\
0
\end{array}\right],\left[\begin{array}{l}
0 \\
0 \\
1 \\
0
\end{array}\right],\left[\begin{array}{l}
0 \\
0 \\
0 \\
1
\end{array}\right]\right\} .
$$

On the other hand, if $\Gamma_{2}$ is the chosen chamber, then

$$
\mathcal{K}=\mathbb{N}\left\{\left[\begin{array}{l}
0 \\
2 \\
0 \\
0
\end{array}\right],\left[\begin{array}{l}
2 \\
2 \\
0 \\
0
\end{array}\right]\right\} \quad \text { and } \quad \mathcal{K}^{\text {sat }}=\mathbb{N}\left\{\left[\begin{array}{l}
0 \\
1 \\
0 \\
0
\end{array}\right],\left[\begin{array}{l}
1 \\
1 \\
0 \\
0
\end{array}\right],\left[\begin{array}{l}
0 \\
0 \\
1 \\
0
\end{array}\right],\left[\begin{array}{l}
0 \\
0 \\
0 \\
1
\end{array}\right]\right\} .
$$

The following lemma further illustrates the connections between the ideal $B$ and the semigroups $\mathcal{K}$ and $\mathcal{K}^{\text {sat }}$. For $\boldsymbol{p} \in G$, let $\left\langle S_{\boldsymbol{p}}\right\rangle$ denote the ideal generated by all the monomials of degree $\boldsymbol{p}$ in $S$. For $\boldsymbol{u} \in \mathbb{N}^{n}$, let $\operatorname{supp}(\boldsymbol{u}):=\left\{i: u_{i} \neq 0\right\} \subseteq[n]$.

Lemma 2.4. If $\boldsymbol{p}$ belongs to the interior of $\mathcal{K}^{\mathrm{sat}}$ and $\operatorname{deg}\left(\boldsymbol{x}^{\boldsymbol{u}}\right)=\boldsymbol{p}$ then $\boldsymbol{x}^{\boldsymbol{u}}$ belongs to the ideal $B$. Moreover, if $\boldsymbol{p} \in \mathcal{K}$, then $B \subseteq \sqrt{\left\langle S_{\boldsymbol{p}}\right\rangle}$.

Proof. If $\boldsymbol{p} \in \operatorname{int} \mathcal{K}^{\text {sat }}$ and $\boldsymbol{x}^{\boldsymbol{u}} \in S_{\boldsymbol{p}}$ then $\operatorname{pos}\left(\overline{\boldsymbol{a}}_{i}: i \in \operatorname{supp}(\boldsymbol{u})\right) \cap \operatorname{int} \Gamma \neq \emptyset$. Since $\Gamma$ is a maximal cell in the chamber complex of $\overline{\mathcal{A}}$, we have $\Gamma \subseteq \operatorname{pos}\left(\overline{\boldsymbol{a}}_{i}: i \in \operatorname{supp}(\boldsymbol{u})\right)$ and $\operatorname{dim}_{\mathbb{R}} \operatorname{pos}\left(\overline{\boldsymbol{a}}_{i}: i \in \operatorname{supp}(\boldsymbol{u})\right)=r$. Caratheodory's Theorem (Proposition 1.15 in Zie] implies that $\operatorname{pos}\left(\overline{\boldsymbol{a}}_{i}: i \in \operatorname{supp}(\boldsymbol{u})\right)$ is the union of the $\operatorname{pos}\left(\overline{\boldsymbol{a}}_{i}: i \in \sigma\right)$ where $|\sigma|=r$ and $\sigma \subseteq \operatorname{supp}(\boldsymbol{u})$. Hence, there exists $\sigma \subseteq \operatorname{supp}(\boldsymbol{u})$ such that $|\sigma|=r$ and $\operatorname{pos}\left(\overline{\boldsymbol{a}}_{i}: i \in \sigma\right) \cap$ int $\Gamma \neq \emptyset$. Again, because $\Gamma$ is a chamber, we have $\Gamma \subseteq \operatorname{pos}\left(\overline{\boldsymbol{a}}_{i}: i \in \sigma\right)$. It follows that the monomial $\prod_{i \in \sigma} x_{i}$ belongs to $B$ and divides $\boldsymbol{x}^{\boldsymbol{u}}$. This establishes the first assertion. 
If $\boldsymbol{p} \in \mathcal{K}$, then for every $\sigma \in \Delta$ there exists a monomial $\boldsymbol{x}^{\boldsymbol{u}} \in S_{\boldsymbol{p}}$ with $\operatorname{supp}(\boldsymbol{u}) \subseteq \widehat{\sigma}$. It follows that a sufficiently large power of each generator of $B$ belongs to the ideal $\left\langle S_{\boldsymbol{p}}\right\rangle$ which implies $B \subseteq \sqrt{\left\langle S_{\boldsymbol{p}}\right\rangle}$.

Our motivating example of a pair $(S, B)$ is the homogeneous coordinate ring and irrelevant ideal of a toric variety introduced in Co2 . Let $X$ be a simplicial toric variety over a field $\mathbb{k}$ determined by a fan $\Delta$ in $\mathbb{R}^{d}$. By numbering the rays (one-dimensional cones) of $\Delta$, we identify $\Delta$ with a simplicial complex on $[n]$. We write $\boldsymbol{b}_{1}, \ldots, \boldsymbol{b}_{n}$ for the unique minimal lattice vectors generating the rays and we assume that the positive hull of $\mathcal{B}:=\left\{\boldsymbol{b}_{1}, \ldots, \boldsymbol{b}_{n}\right\}$ is $\mathbb{R}^{d}$. The set $\mathcal{B}$ gives rise to a short exact sequence

$$
0 \longrightarrow \mathbb{Z}^{d} \stackrel{\left[\boldsymbol{b}_{1} \cdots \boldsymbol{b}_{n}\right]^{\top}}{\longrightarrow} \mathbb{Z}^{n} \longrightarrow G \longrightarrow 0 .
$$

Tensoring this sequence with $\mathbb{R}$, we obtain (2.0.1). Geometrically, $G$ is the divisor class group (or Chow group) of $X$; see $\S 3.4$ in [Ful]. The image of the $i$ th standard basis vector of $\mathbb{Z}^{n}$ in $G$ is denoted by $\boldsymbol{a}_{i}$. Observe that the set $\mathcal{A}=\left\{\boldsymbol{a}_{1}, \ldots, \boldsymbol{a}_{n}\right\}$ is uniquely determined up to an automorphism of $G$. The homogeneous coordinate ring of $X$ is the polynomial ring $S=\mathbb{k}\left[x_{1}, \ldots, x_{n}\right]$ with the $G$-grading induced by $\operatorname{deg}\left(x_{i}\right)=\boldsymbol{a}_{i}$ and the irrelevant ideal is $B=\left\langle\prod_{i \notin \sigma} x_{i}: \sigma \in \Delta\right\rangle$.

This geometric choice of a pair $(S, B)$ fits into the algebraic framework developed at the start of this section if the fan of $X$ corresponds to a regular triangulation of $\mathcal{B}$. By Theorem 2.6 in [HtS, this is equivalent to saying that $X$ is semi-projective. In particular, this holds whenever $X$ is projective. Conversely, an algebraic pair $(S, B)$ is the homogeneous coordinate ring and irrelevant ideal of a simplicial toric variety if and only if, for each $i$, the ray $\operatorname{pos}\left(\overline{\boldsymbol{b}}_{i}\right)$ belongs to the triangulation associated to the chamber $\Gamma$.

Example 2.5. The pair $(S, B)$ described in Example 2.1 corresponds to the weighted projective space $X=\mathbb{P}\left(\boldsymbol{a}_{1}, \ldots, \boldsymbol{a}_{n}\right)$. In particular, when $X=\mathbb{P}^{d}$, the homogeneous coordinate ring $S$ is the standard graded polynomial ring and the irrelevant ideal $B$ is the unique graded maximal ideal.

Example 2.6. If $(S, B)$ is the pair described in Example 2.2 where $\Gamma=\mathbb{R}_{\geq 0}^{2}$ then the associated toric variety is the Hirzebruch surface (or rational scroll) $\mathbb{F}_{t}=\mathbb{P}\left(\mathscr{O}_{\mathbb{P}^{1}} \oplus\right.$ $\left.\mathscr{O}_{\mathbb{P}^{1}}(t)\right)$.

Example 2.7. If $X$ is the product of projective space $\mathbb{P}^{d} \times \mathbb{P}^{e}$, then the homogeneous coordinate ring $S=\mathbb{k}\left[x_{0}, \ldots, x_{d}, y_{0}, \ldots, y_{e}\right]$ has the $\mathbb{Z}^{2}$-grading induced by $\operatorname{deg}\left(x_{i}\right)=\left[\begin{array}{l}1 \\ 0\end{array}\right]$ and $\operatorname{deg}\left(y_{i}\right)=\left[\begin{array}{l}0 \\ 1\end{array}\right]$ and the irrelevant ideal $B$ is $\left\langle x_{0}, \ldots, x_{d}\right\rangle \cap\left\langle y_{0} \ldots, y_{e}\right\rangle$.

When the pair $(S, B)$ corresponds to a simplicial toric variety $X$, the chamber $\Gamma$ and the semigroups $\mathcal{K}$ and $\mathcal{K}^{\text {sat }}$ have geometric interpretations. Assuming all the maximal cones are $d$-dimensional, we have $\operatorname{Pic}(X) \otimes \mathbb{Q} \cong G \otimes \mathbb{Q}$. As $\S 3$ in Co3] indicates, the chamber $\Gamma$ is the closure of the Kähler cone of $X$. The dual of the Kähler cone is the Mori cone of effective 1-cycles modulo numerical equivalence. The semigroup $\mathcal{K}^{\text {sat }}$ corresponds to the numerically effective Weil divisors on $X$ up to rational equivalence and elements in $\mathcal{K}$ are nef line bundles. When $X$ is smooth, the class group $G$ is torsion-free and $\mathcal{K}=\mathcal{K}^{\text {sat }}$.

The next example demonstrates that $\mathcal{K}$ can be complicated even when $X$ is smooth. 
Example 2.8. Consider the following smooth resolution $X$ of weighted projective space $\mathbb{P}(2,3,7,1)$. Specifically, the set $\mathcal{B}$ corresponds to the columns of the matrix

$$
\left[\begin{array}{ccccccccccc}
1 & 0 & 0 & -2 & 0 & 0 & -1 & 0 & -1 & -1 & -1 \\
0 & 1 & 0 & -3 & 0 & -1 & -1 & -1 & -1 & -2 & -2 \\
0 & 0 & 1 & -7 & -1 & -2 & -3 & -3 & -4 & -4 & -5
\end{array}\right] .
$$

Figure 3 illustrates the regular triangulation and the irrelevant ideal is

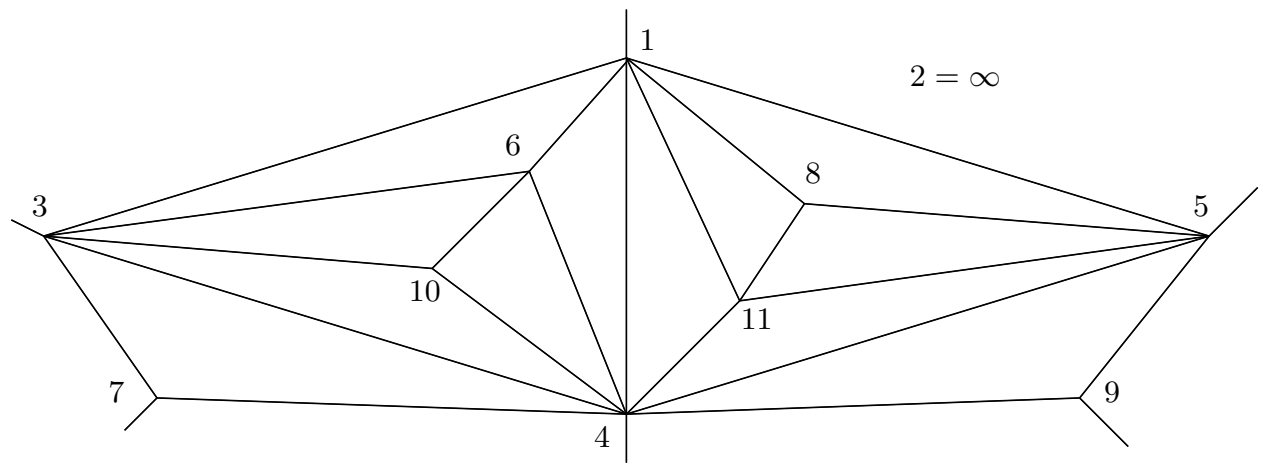

Figure 3. The triangulation for Example 2.8

$$
\begin{array}{rlll}
B=\left\langle x_{1} x_{2} x_{3} x_{4} x_{6} x_{7} x_{9} x_{10},\right. & x_{2} x_{3} x_{4} x_{5} x_{6} x_{7} x_{9} x_{10}, & x_{1} x_{2} x_{3} x_{6} x_{7} x_{8} x_{9} x_{10}, \\
& x_{2} x_{3} x_{5} x_{6} x_{7} x_{8} x_{9} x_{10}, & x_{1} x_{2} x_{3} x_{5} x_{7} x_{8} x_{9} x_{11}, & x_{1} x_{2} x_{4} x_{5} x_{7} x_{8} x_{9} x_{11}, \\
& x_{1} x_{2} x_{5} x_{6} x_{7} x_{8} x_{9} x_{11}, & x_{1} x_{2} x_{3} x_{6} x_{7} x_{8} x_{10} x_{11}, & x_{1} x_{3} x_{4} x_{6} x_{7} x_{8} x_{10} x_{11}, \\
& x_{1} x_{3} x_{5} x_{6} x_{7} x_{8} x_{10} x_{11}, & x_{2} x_{3} x_{4} x_{6} x_{7} x_{9} x_{10} x_{11}, & x_{1} x_{2} x_{5} x_{6} x_{8} x_{9} x_{10} x_{11}, \\
& x_{1} x_{3} x_{5} x_{6} x_{8} x_{9} x_{10} x_{11}, & x_{1} x_{4} x_{5} x_{6} x_{8} x_{9} x_{10} x_{11}, & x_{2} x_{3} x_{5} x_{7} x_{8} x_{9} x_{10} x_{11}, \\
& x_{2} x_{4} x_{5} x_{7} x_{8} x_{9} x_{10} x_{11}, & x_{3} x_{4} x_{6} x_{7} x_{8} x_{9} x_{10} x_{11}, & \left.x_{4} x_{5} x_{6} x_{7} x_{8} x_{9} x_{10} x_{11}\right\rangle .
\end{array}
$$

In this case, $\Gamma$ is an 8-dimensional cone with 16 extremal rays. Using [BK], we determine that set of minimal generators for $\mathcal{K}$ has 25 elements all of which lie on the boundary of $\Gamma$.

\section{A Topological Formula for local Cohomology}

Throughout this paper, $M$ denotes a finitely generated $G$-graded $S$-module. In this section we derive a vanishing theorem for the local cohomology modules $H_{B}^{i}(M)$ from a topological formula for $H_{B}^{i}(S)$. We refer to $\mathrm{BrS}$ ] for background information on local cohomology. A module $M$ is $B$-torsion if $M=H_{B}^{0}(M)$. If $M$ is $B$-torsion then $H_{B}^{i}(M)=0$ for $i>0$. For an element $g \in S$, we set

$$
\left(0:_{M} g\right):=\{f \in M: g f=0\}=\operatorname{Ker}(M \stackrel{g}{\longrightarrow} M) .
$$

This submodule is zero when $g$ is a nonzerodivisor on $M$. We say an element $g \in S$ is almost a nonzerodivisor on $M$ if $\left(0:_{M} g\right)$ is a $B$-torsion module.

Proposition 3.1. Let $\mathbb{k}$ be an infinite field. If $\boldsymbol{p} \in \mathcal{K}$ and $g \in S$ is a sufficiently general form of degree $\boldsymbol{p}$, then $g$ is almost a nonzerodivisor on $M$. In other words, there is an open dense set of degree $\boldsymbol{p}$ forms $g$ for which $\left(0:_{M} g\right)$ is B-torsion. 
Proof. The module $M^{\prime}:=\left(0:_{M} g\right)$ is $B$-torsion if each element in $M^{\prime}$ is annihilated by some power of $B$. This is equivalent to saying that the localization $M_{P}^{\prime}=0$ for all prime ideals $P$ in $S$ not containing $B$. In other words, $g$ is a nonzerodivisor on the localization $M_{P}$. Hence, it suffices to show that $g$ is not contained in any of the associated primes of $M$ except possibly those which contain $B$.

To accomplish this, observe that each prime ideal $P$ in $S$ which does not contain $B$ intersects $S_{\boldsymbol{p}}$ in a proper subspace. Otherwise $\left\langle S_{\boldsymbol{p}}\right\rangle \subseteq P$ and Lemma 2.4 implies that $B \subseteq \sqrt{\left\langle S_{\boldsymbol{p}}\right\rangle} \subseteq \sqrt{P}=P$ which contradicts the hypothesis on $P$. Because $M$ has only finitely many associated primes, our observation shows that $g \in S_{\boldsymbol{p}}$ is almost a nonzerodivisor on $M$ if it lies outside a certain finite union of proper subspaces. Since $\mathbb{k}$ is infinite, the vector space $S_{\boldsymbol{p}}$ is not a finite union of proper subspaces.

To give a uniform vanishing result for local cohomology, we assume for the remainder of this section that $\operatorname{pos}(\overline{\mathcal{A}})$ is a pointed cone with $\overline{\boldsymbol{a}}_{i} \neq \mathbf{0}$ for each $i$ or equivalently that $\overline{\mathcal{A}}$ is an acyclic vector configuration; see $\S 6.2$ in [Zie]. This condition holds if $S$ is the homogeneous coordinate ring of a projective toric variety. We can rephrase this assumption by saying that the dual configuration $\overline{\mathcal{B}}$ is totally cyclic or $\operatorname{pos}(\overline{\mathcal{B}})=\mathbb{R}^{d}$. Hence, any regular triangulation of $\overline{\mathcal{B}}$ is a complete fan in $\mathbb{R}^{d}$. We may identify $\Delta$ with a simplicial $(d-1)$-sphere and the collection $\widehat{\Delta}:=\Delta \cup\{[n]\}$ (ordered by inclusion) with the face poset of a finite regular cell decomposition of a $d$-ball. It follows that there is an incidence function $\varepsilon$ on $\widehat{\Delta}$; see $\S 6.2$ in $[\mathrm{BH}]$.

For $\sigma \subseteq[n]$, let $\boldsymbol{x}_{\sigma}$ denote the squarefree monomial $\prod_{i \in \sigma} x_{i} \in S$. The canonical $\check{C} e c h$ complex associated to $\widehat{\Delta}$ is the following chain complex:

$$
\check{\mathbf{C}}:=0 \longrightarrow \check{C}^{0} \stackrel{\partial^{0}}{\longrightarrow} \check{C}^{1} \stackrel{\partial^{1}}{\longrightarrow} \check{C}^{2} \stackrel{\partial^{2}}{\longrightarrow} \cdots \stackrel{\partial_{d-1}}{\longrightarrow} \check{C}^{d} \stackrel{\partial_{d}}{\longrightarrow} \check{C}^{d+1} \longrightarrow 0,
$$

where $\check{C}^{0}:=S, \check{C}^{i}:=\bigoplus_{\sigma \in \Delta,|\sigma|=d+1-i} S\left[\boldsymbol{x}_{\widehat{\sigma}}^{-1}\right]$ for $i>0$ and $\partial^{i}: \check{C}^{i} \longrightarrow \check{C}^{i+1}$ is composed of homomorphisms $\varepsilon(\sigma, \tau) \cdot$ nat: $S\left[\boldsymbol{x}_{\widehat{\sigma}}^{-1}\right] \longrightarrow S\left[\boldsymbol{x}_{\widehat{\tau}}^{-1}\right]$. By combining Corollary 2.13 in [BbS and Theorem 6.2 in [Mil, we deduce that $H_{B}^{i}(M)=H^{i}\left(\check{\mathbf{C}} \otimes_{S} M\right)$ for all $S$ modules $M$. It follows that $H_{B}^{i}(M)=0$ for all $i<0$ or $i>d+1$.

The next proposition gives a combinatorial description for the local cohomology of the polynomial ring $S$. We will use this formula to compute the multigraded regularity in some examples. This result is a variant on the formulae found in [EMS] and [RWY]. For $\sigma \in \Delta$, let $\Delta_{\sigma}$ be the induced subcomplex $\{\tau \in \Delta: \tau \subseteq \sigma\}$ of $\Delta$. The modules $H_{B}^{i}(S)$ have a $\mathbb{Z}^{n}$-grading which refines their $G$-grading.

Proposition 3.2. Let $\widetilde{H}^{i}(-)$ denote the ith reduced cellular cohomology group with coefficients in $\mathbb{k}$. If $\boldsymbol{u} \in \mathbb{Z}^{n}$ and $\sigma:=\operatorname{neg}(\boldsymbol{u})=\left\{j: \boldsymbol{u}_{j}<0\right\}$, then we have

$$
H_{B}^{i}(S)_{\boldsymbol{u}} \cong \begin{cases}\widetilde{H}^{i-2}\left(\Delta_{\sigma}\right) & \text { for } i \neq 1 \\ 0 & \text { for } i=1\end{cases}
$$

Proof. One easily checks (see Lemma 5.3.6 in $[\mathrm{BH}]$ ) that $\operatorname{dim}_{\mathbb{k}} S\left[\boldsymbol{x}_{\widehat{\tau}}^{-1}\right]_{\boldsymbol{u}}=1$ when $\tau \subseteq \widehat{\sigma}$ and $\operatorname{dim}_{\mathbb{k}} S\left[\boldsymbol{x}_{\widehat{\tau}}^{-1}\right]_{\boldsymbol{u}}=0$ when $\tau \nsubseteq \widehat{\sigma}$. It follows that the $\boldsymbol{u}$ th graded component of $\check{\mathbf{C}}$ is isomorphic to a shift of the augmented oriented chain complex of $\widehat{\Delta}_{\widehat{\sigma}}$ (see $\S 6.2$ in $[\mathrm{BH}]$ ). More precisely, we have $H^{i}\left(\check{\mathbf{C}}_{\boldsymbol{u}}\right)=\widetilde{H}_{d-i}\left(\widehat{\Delta}_{\widehat{\sigma}}\right)$. To complete the proof, we analyze three cases: 
- If $\sigma$ is a proper nonempty subset of $[n]$, then the subcomplex $\widehat{\Delta}_{\widehat{\sigma}}$ equals $\Delta_{\widehat{\sigma}}$. Alexander duality (Theorem 71.1 in Mun] shows that $\widetilde{H}_{d-i}\left(\Delta_{\widehat{\sigma}}\right)$ is isomorphic to $\widetilde{H}^{i-2}\left(\Delta \backslash \Delta_{\widehat{\sigma}}\right)$. Because $\Delta_{\sigma}$ is a deformation retract of $\Delta \backslash \Delta_{\widehat{\sigma}}$ (Lemma 70.1 in Mun], we have $\widetilde{H}_{d-i}\left(\Delta_{\widehat{\sigma}}\right) \cong \widetilde{H}^{i-2}\left(\Delta_{\sigma}\right)$. Note that $\widetilde{H}^{i-2}\left(\Delta_{\sigma}\right)=0$ when $i=1$.

- If $\sigma=\emptyset$, then $\widehat{\sigma}=[n]$ and $\widehat{\Delta}_{\widehat{\sigma}}$ is a $d$-ball which implies that $\widetilde{H}_{d-i}\left(\widehat{\Delta}_{\widehat{\sigma}}\right)=0$ for all $i$. It follows that $\widetilde{H}_{d-i}\left(\widehat{\Delta}_{[n]}\right)$ is isomorphic to $\widetilde{H}^{i-2}\left(\Delta_{\emptyset}\right)$ for $i \neq 1$ and equals 0 for $i=1$.

- If $\sigma=[n]$, then $\widehat{\sigma}=\emptyset$ and $\widehat{\Delta}_{\widehat{\sigma}}=\emptyset$ which implies that $\widetilde{H}_{d-i}\left(\widehat{\Delta}_{\widehat{\sigma}}\right)=0$ for $i \neq d+1$ and $\widetilde{H}_{-1}\left(\widehat{\Delta}_{\widehat{\sigma}}\right) \cong \mathbb{k}$. Since $\Delta_{[n]}=\Delta$ is a $(d-1)$-sphere, we also have $\widetilde{H}_{d-i}\left(\widehat{\Delta}_{\emptyset}\right) \cong \widetilde{H}^{i-2}\left(\Delta_{[n]}\right)$ for all $i$.

Remark 3.3. Proposition 3.2 implies that $H_{B}^{i}(S) \neq 0$ only if $2 \leq i \leq d+1$. Since $H_{B}^{d+1}(S)_{\boldsymbol{u}}=\mathbb{k}$ when $\operatorname{neg}(\boldsymbol{u})=[n]$, we have $H_{B}^{d+1}(S) \neq 0$. Moreover, $H_{B}^{d+1}(S)$ is the only nonvanishing local cohomology module if and only if every proper subcomplex of $\Delta$ is contractible. This happens precisely when $\Delta$ is the boundary of the standard simplex. Hence, $H_{B}^{i}(S)=0$ for all $i \neq d+1$ if and only if $B=\left\langle x_{1}, \ldots, x_{n}\right\rangle$.

Using Proposition 3.2, we can describe the Hilbert series of the modules $H_{B}^{i}(S)$.

Corollary 3.4. For all $i \neq 1$, we have

$$
\sum_{\boldsymbol{p} \in G} \operatorname{dim}_{\mathbb{k}} H_{B}^{i}(S)_{\boldsymbol{p}} \cdot \boldsymbol{t}^{\boldsymbol{p}}=\sum_{\sigma \subseteq[n]} \frac{\operatorname{dim}_{\mathbb{k}} \widetilde{H}^{i-2}\left(\Delta_{\sigma}\right) \cdot \prod_{j \in \sigma} \boldsymbol{t}^{-\boldsymbol{a}_{j}}}{\prod_{j \in \widehat{\sigma}}\left(1-\boldsymbol{t}^{\boldsymbol{a}_{j}}\right) \prod_{j \in \sigma}\left(1-\boldsymbol{t}^{-\boldsymbol{a}_{j}}\right)} .
$$

Proof. For $i \neq 1$, Proposition 3.2 implies that

$$
\begin{aligned}
\sum_{\boldsymbol{u} \in \mathbb{Z}^{n}} \operatorname{dim}_{\mathbb{k}} H_{B}^{i}(S)_{\boldsymbol{u}} \cdot \boldsymbol{x}^{\boldsymbol{u}}=\sum_{\boldsymbol{u} \in \mathbb{Z}^{n}} \operatorname{dim}_{\mathbb{k}} \widetilde{H}^{i-2}\left(\Delta_{\mathrm{neg}(\boldsymbol{u})}\right) \cdot \boldsymbol{x}^{\boldsymbol{u}} \\
=\sum_{\sigma \subseteq[n]}\left(\operatorname{dim}_{\mathbb{k}} \widetilde{H}^{i-2}\left(\Delta_{\sigma}\right) \sum_{\operatorname{neg}(\boldsymbol{u})=\sigma} \boldsymbol{x}^{\boldsymbol{u}}\right)=\sum_{\sigma \subseteq[n]} \frac{\operatorname{dim}_{\mathbb{k}} \widetilde{H}^{i-2}\left(\Delta_{\sigma}\right) \cdot \prod_{j \in \sigma} x_{j}^{-1}}{\prod_{j \in \widehat{\sigma}}\left(1-x_{j}\right) \prod_{j \in \sigma}\left(1-x_{j}^{-1}\right)} .
\end{aligned}
$$

Mapping $x_{j}$ to $\boldsymbol{t}^{\boldsymbol{a}_{j}}$ establishes the corollary.

We illustrate this corollary with the following example.

Example 3.5. If $S$ is the homogeneous coordinate ring of the Hirzebruch surface $\mathbb{F}_{t}$ as in Examples 2.2] \& 2.6. then the only non-contractible subcomplexes are $\Delta_{\emptyset}, \Delta_{\{1,3\}}, \Delta_{\{2,4\}}$ and $\Delta_{[4]}$. Since $\widetilde{H}^{i}\left(\Delta_{\emptyset}\right)=0$ for all $i \neq-1$ and $\widetilde{H}^{-1}\left(\Delta_{\emptyset}\right)=\mathbb{k}$, this subcomplex does not contribute to any local cohomology module. Because $\widetilde{H}^{0}\left(\Delta_{\{1,3\}}\right)=\mathbb{k}=\widetilde{H}^{0}\left(\Delta_{\{2,4\}}\right)$, the degrees $\boldsymbol{p} \in \mathbb{Z}^{2}$ for which $H_{B}^{2}(S)_{\boldsymbol{p}} \neq 0$ correspond to the lattices points in the two cones $-\boldsymbol{a}_{1}-\boldsymbol{a}_{3}+\operatorname{pos}\left(-\boldsymbol{a}_{1}, \boldsymbol{a}_{2},-\boldsymbol{a}_{3}, \boldsymbol{a}_{4}\right)$ and $-\boldsymbol{a}_{2}-\boldsymbol{a}_{4}+\operatorname{pos}\left(\boldsymbol{a}_{1},-\boldsymbol{a}_{2}, \boldsymbol{a}_{3},-\boldsymbol{a}_{4}\right)$. We also have $\widetilde{H}^{1}\left(\Delta_{[n]}\right)=\mathbb{k}$, so the degrees $\boldsymbol{p} \in \mathbb{Z}^{2}$ for which $H_{B}^{2}(S)_{\boldsymbol{p}} \neq 0$ correspond to the lattices points in the cone $-\boldsymbol{a}_{1}-\boldsymbol{a}_{2}-\boldsymbol{a}_{3}-\boldsymbol{a}_{4}+\operatorname{pos}\left(-\boldsymbol{a}_{1},-\boldsymbol{a}_{2},-\boldsymbol{a}_{3},-\boldsymbol{a}_{4}\right)$. These cones are indicated by the shaded areas in Figure 4 when $t=2$.

The next result extends a well-known vanishing theorem for ample line bundles on a complete toric variety; see (6.3.1) for the explicit connection. 

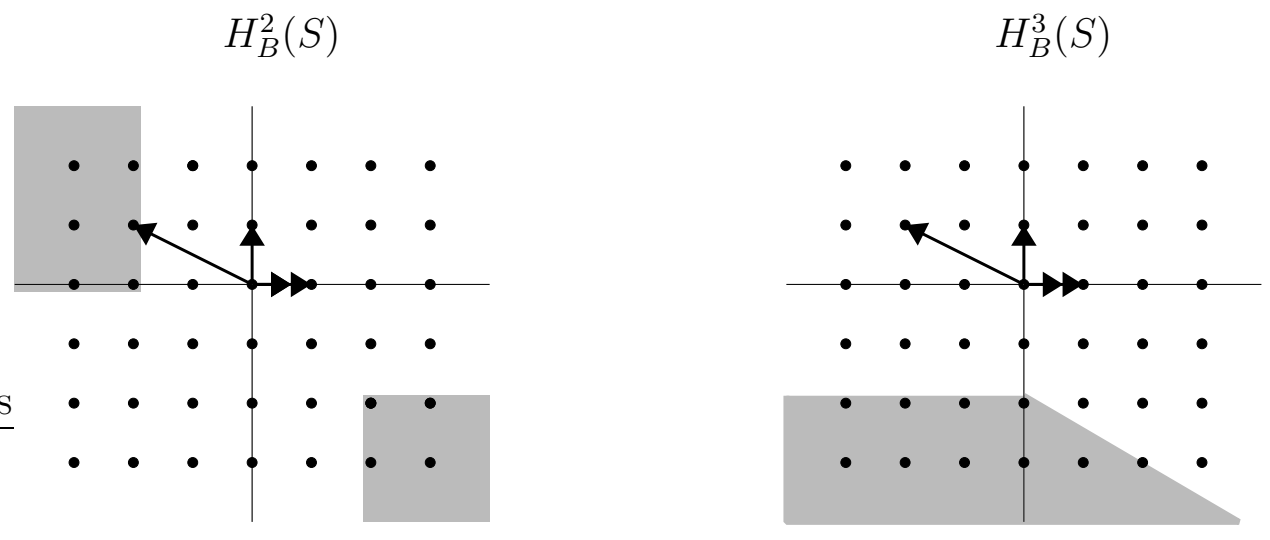

FiguRE 4. Degrees $\boldsymbol{p} \in \mathbb{Z}^{2}$ for which $H_{B}^{i}(S)_{\boldsymbol{p}} \neq 0$ in Example 3.5.

Corollary 3.6. If $\boldsymbol{p}$ belongs to $\mathcal{K}^{\mathrm{sat}}$, then $H_{B}^{i}(S)_{\boldsymbol{p}}$ vanishes.

Proof. Fix $\boldsymbol{p} \in \mathcal{K}^{\text {sat }}$ and suppose $\boldsymbol{u} \in \mathbb{Z}^{n}$ satisfies $\operatorname{deg}\left(\boldsymbol{x}^{\boldsymbol{u}}\right)=\boldsymbol{p}$. The vector $\boldsymbol{u}$ defines a function on $\psi_{\boldsymbol{u}}: \mathbb{R}^{d} \longrightarrow \mathbb{R}$ that is linear on the cone $\operatorname{pos}\left(\overline{\boldsymbol{b}}_{i}: i \in \sigma\right)$ for all $\sigma \in \Delta$ and satisfies $\psi_{\boldsymbol{u}}\left(\overline{\boldsymbol{b}}_{i}\right)=-u_{i}$ for $1 \leq i \leq n$. Lemma 3.2 in BGS implies that $\boldsymbol{p} \in \mathcal{K}^{\text {sat }}$ if and only if $\psi_{\boldsymbol{u}}$ is convex which means $\psi_{\boldsymbol{u}}\left(\boldsymbol{w}+\boldsymbol{w}^{\prime}\right) \geq \psi_{\boldsymbol{u}}(\boldsymbol{w})+\psi_{\boldsymbol{u}}\left(\boldsymbol{w}^{\prime}\right)$ for all $\boldsymbol{w}$, $\boldsymbol{w}^{\prime} \in \mathbb{R}^{d}$. Hence, the set $\left\{\boldsymbol{w} \in \mathbb{R}^{d}: \psi_{\boldsymbol{u}}(\boldsymbol{w}) \geq 0\right\}$ is convex. It follows that simplicial complex $\Delta_{\text {neg }(\boldsymbol{u})}$ corresponds to the intersection of a convex set with the $(d-1)$-sphere. Therefore, $\Delta_{\text {neg }(\boldsymbol{u})}$ is contractible and Proposition 3.2 implies that $H_{B}^{i}(S)_{\boldsymbol{p}}=0$ which proves the statement.

As a further corollary, we obtain a vanishing theorem for the local cohomology of any finitely generated $G$-graded $S$-module. Geometrically, this result corresponds to Fujita's vanishing theorem (Theorem 1.4.32 in [Laz]). We first record a useful observation.

Remark 3.7. Let $\mathcal{D}$ be a subsemigroup of $G$ consisting of all points $\boldsymbol{p} \in G$ such that their image $\overline{\boldsymbol{p}}$ in $\mathbb{R}^{r}$ lies in a fixed convex cone $C$. If $\boldsymbol{p}+\mathcal{D}$ and $\boldsymbol{q}+\mathcal{D}$ are two shifts of $\mathcal{D}$, then their intersection is nonempty. In fact, if $\boldsymbol{p} \in \mathcal{D}$, with $\overline{\boldsymbol{p}} \in \operatorname{int} C$, then for all sufficiently large $j \in \mathbb{N}$ we have $j \boldsymbol{p} \in \boldsymbol{q}+\mathcal{D}$ for any $\boldsymbol{q} \in G$.

Corollary 3.8. There is $\boldsymbol{m} \in \mathcal{K}^{\text {sat }}$ such that $H_{B}^{i}(M)_{\boldsymbol{p}}=0$ for all $i$ and all $\boldsymbol{p} \in \boldsymbol{m}+\mathcal{K}^{\text {sat }}$. In particular, $H_{B}^{i}(M)_{\boldsymbol{p}}$ vanishes for all $i$ and all $\boldsymbol{p} \in \mathcal{K}^{\text {sat }}$ sufficiently far into the interior of $\mathcal{K}^{\text {sat }}$. If desired we may assume $\boldsymbol{m} \in \mathcal{K}$.

Proof. We proceed by induction on the projective dimension $\operatorname{pd}(M)$ of $M$. Since $M$ is finitely generated, there is a short exact sequence $0 \longrightarrow E_{1} \longrightarrow E_{0} \longrightarrow M \longrightarrow 0$ where $E_{0}=\bigoplus_{1 \leq j \leq h} S\left(-\boldsymbol{q}_{j}\right)$ for some $\boldsymbol{q}_{j} \in G$. The associated long exact sequence contains

$$
H_{B}^{i}\left(E_{0}\right)_{\boldsymbol{p}} \longrightarrow H_{B}^{i}(M)_{\boldsymbol{p}} \longrightarrow H_{B}^{i+1}\left(E_{1}\right)_{\boldsymbol{p}}
$$

If we choose $\boldsymbol{m}^{\prime} \in \bigcap_{1 \leq j \leq h}\left(\boldsymbol{q}_{j}+\mathcal{K}^{\text {sat }}\right)$, which is possible by Remark 3.7, then Theorem 3.6 implies that the left module in (3.8.2) vanishes for all $i$ and all $\boldsymbol{p} \in \boldsymbol{m}^{\prime}+\mathcal{K}^{\text {sat }}$. When $\operatorname{pd}(M)=0$, we have $E_{1}=0$ and there is nothing more to prove. Otherwise, we have $E_{1} \neq 0$ and $\operatorname{pd}\left(E_{1}\right)<\operatorname{pd}(M)$. Hence, the induction hypothesis provides $\boldsymbol{m}^{\prime \prime} \in G$ such that the right module in (3.8.2) vanishes for all $\boldsymbol{p} \in \boldsymbol{m}^{\prime \prime}+\mathcal{K}^{\text {sat }}$. Therefore, by choosing 
$\boldsymbol{m} \in\left(\boldsymbol{m}^{\prime}+\mathcal{K}^{\text {sat }}\right) \cap\left(\boldsymbol{m}^{\prime \prime}+\mathcal{K}^{\text {sat }}\right)$, which is again possible by Remark 3.7, we see that the middle module in (3.8.2) vanishes for all $i$ and all $\boldsymbol{p} \in \boldsymbol{m}+\mathcal{K}^{\text {sat }}$.

Lastly, because $\mathcal{K}^{\text {sat }}$ is the saturation of $\mathcal{K}$, there exists some $\boldsymbol{m}^{\prime \prime \prime} \in \mathcal{K} \cap\left(\boldsymbol{m}+\mathcal{K}^{\text {sat }}\right)$. Since $\boldsymbol{m}^{\prime \prime \prime}+\mathcal{K}^{\text {sat }} \subseteq \boldsymbol{m}+\mathcal{K}^{\text {sat }}$, the corresponding vanishing statement holds for $\boldsymbol{m}^{\prime \prime \prime}$ which establishes the last part of the corollary.

We end this section by examining the assumption that $\operatorname{pos}(\overline{\mathcal{A}})$ is acyclic. Firstly, we can remove the condition that $\boldsymbol{a}_{i} \neq \mathbf{0}$ for all $i$.

Remark 3.9. Let $\sigma:=\left\{i \in[n]: \overline{\boldsymbol{a}}_{i}=\mathbf{0}\right\}$. For each $j \in \sigma$ the variable $x_{j}$ does not divide any minimal generator of $B$. In other words, the set $\sigma$ is contained in every facet of $\Delta$. Hence, the simplicial complex $\Delta$ is a cone over the induced subcomplex $\Delta_{\widehat{\sigma}}$. By replacing $\Delta$ with $\Delta_{\widehat{\sigma}}$, we can extend Proposition 3.2 and its corollaries to this more general situation.

On the other hand, the next example shows that we cannot eliminate the hypothesis that $\operatorname{pos}(\overline{\mathcal{A}})$ is a pointed cone.

Example 3.10. If $G=\mathbb{Z}$ and $\mathcal{A}=\{\mathbf{1}, \mathbf{- 1}, \mathbf{1}\}$, then $S=\mathbb{k}\left[x_{1}, x_{2}, x_{3}\right]$ has the $\mathbb{Z}$-grading induced by $\operatorname{deg}\left(x_{2}\right)=\mathbf{- 1}$ and $\operatorname{deg}\left(x_{1}\right)=\operatorname{deg}\left(x_{3}\right)=\mathbf{1}$. Choosing the chamber $\Gamma$ to be $\mathbb{R}_{\geq 0}$, we have $\mathcal{K}=\mathcal{K}^{\text {sat }}=\mathbb{N}$. The corresponding monomial ideal is $B=\left\langle x_{1}, x_{3}\right\rangle$ and the dual vector configuration corresponds to the columns of the matrix $\left[\begin{array}{lll}1 & 1 & 0 \\ 0 & 1 & 1\end{array}\right]$. Since the first paragraph of the proof of Proposition 3.2 applies in this situation, $H_{B}^{2}(S)_{\boldsymbol{u}}=\widetilde{H}_{0}\left(\widehat{\Delta}_{\widehat{\sigma}}\right)$ where $\sigma=\operatorname{neg}(\boldsymbol{u})$. If $\sigma=\{2\}$, then $\widehat{\sigma}=\{1,3\}$ and $\widehat{\Delta}_{\{1,3\}}=\{\emptyset,\{1\},\{3\}\}$. It follows that $\widetilde{H}_{0}\left(\widehat{\Delta}_{\widehat{\sigma}}\right) \neq 0$ which implies that $H_{B}^{2}(S)_{\boldsymbol{u}} \neq 0$ when $\operatorname{neg}(\boldsymbol{u})=\{2\}$. However, for all $\boldsymbol{p} \in \mathbb{N}$, there exists a $\boldsymbol{u} \in \mathbb{Z}^{3}$ with $\operatorname{neg}(\boldsymbol{u})=\{2\}$. We conclude that $H_{B}^{2}(S)_{\boldsymbol{p}} \neq 0$ for all $p \in \mathcal{K}$.

\section{Regularity of $S$-Modules}

In this section, we define the multigraded regularity of a $G$-graded $S$-module. As in the standard case, multigraded regularity involves the vanishing of certain graded components of local cohomology modules. We show that, in certain cases, the definition is equivalent to an apparently weaker vanishing statement.

Before giving the definition of multigraded regularity, we collect some notation. Throughout this paper, $\mathcal{C}:=\left\{\boldsymbol{c}_{1}, \ldots, \boldsymbol{c}_{\ell}\right\}$ will be a fixed subset of $G$. We write $\mathbb{N} \mathcal{C}$ for the subsemigroup of $G$ generated by $\mathcal{C}$. A subset $\mathcal{D}$ of $G$ is called an $\mathbb{N} \mathcal{C}$-module if for all $\boldsymbol{p} \in \mathcal{D}$ and all $\boldsymbol{q} \in \mathbb{N} \mathcal{C}$ we have $\boldsymbol{p}+\boldsymbol{q} \in \mathcal{D}$. If $\mathcal{D} \subseteq G$ and $i \in \mathbb{Z}$, then $\mathcal{D}[i]:=\bigcup\left(\frac{i}{|i|}\left(\lambda_{1} \boldsymbol{c}_{1}+\cdots+\lambda_{\ell} \boldsymbol{c}_{\ell}\right)+\mathcal{D}\right) \subseteq G$ where the union is over all $\lambda_{1}, \ldots, \lambda_{\ell} \in \mathbb{N}$ such that $\lambda_{1}+\cdots+\lambda_{\ell}=|i|$. For $\boldsymbol{p} \in G$, we clearly have $\boldsymbol{p}+\mathcal{D}[i]=(\boldsymbol{p}+\mathcal{D})[i]$. Moreover, if $\mathcal{D}$ is an $\mathbb{N} \mathcal{C}$-module then $\mathcal{D}[i]$ is also an $\mathbb{N} \mathcal{C}$-module and $\mathcal{D}[i+1] \subseteq \mathcal{D}[i]$. The following definition includes Definition 1.1 as a special case.

Definition 4.1. If $\boldsymbol{m} \in G$, then the $S$-module $M$ is $\boldsymbol{m}$-regular (with respect to $\mathcal{C}$ ) if $H_{B}^{i}(M)_{\boldsymbol{p}}=0$ for all $i \geq 0$ and all $\boldsymbol{p} \in \boldsymbol{m}+\mathbb{N} \boldsymbol{C}[1-i]$. The regularity of $M$, denoted $\operatorname{reg}(M)$, is the set $\{\boldsymbol{m} \in G: M$ is $\boldsymbol{m}$-regular $\}$.

When $S$ has the standard grading, $B=\left\langle x_{1}, \ldots, x_{n}\right\rangle$, and $\mathcal{C}=\{\mathbf{1}\}$, the definition is simply: $M$ is $\boldsymbol{m}$-regular if and only if $H_{B}^{i}(M)_{\boldsymbol{p}}=0$ for all $i \geq 0$ and all $\boldsymbol{p} \geq \boldsymbol{m}-i+1$. 
This is equivalent to the standard definition of Castelnuovo-Mumford regularity; for example see page 282 of $[\mathrm{BrS}]$. Moreover, if $S$ has an $\mathbb{N}$-grading as in Example 2.1] $B=\left\langle x_{1}, \ldots, x_{n}\right\rangle$ and $\mathbf{C}=\{\mathbf{1}\}$, then the definition of $\boldsymbol{m}$-regular is compatible with Definition 4.1 in Ben].

Our definition for $\operatorname{reg}(M)$ conflicts with the standard notation used for CastelnuovoMumford regularity. Traditionally, $G=\mathbb{Z}$ and the regularity of $M$ referred to the smallest $\boldsymbol{m} \in G$ such that $M$ is $\boldsymbol{m}$-regular. In the multigraded setting, the group $G$ is not equipped with a natural ordering so one cannot choose a smallest degree $\boldsymbol{m}$. More importantly, the set $\operatorname{reg}(M)$ may not even be determined by a single element of $G$. Example 4.3 illustrates this phenomenon. For these reasons, we regard the regularity of $M$ as a subset of $G$. From this vantage point, giving a bound on the smallest $\boldsymbol{m}$ such that $M$ is $\boldsymbol{m}$-regular should be interpreted as describing a subset of $\operatorname{reg}(M)$.

Example 4.2. Let $S=\mathbb{k}\left[x_{1}, \ldots, x_{n}\right]$ have the $\mathbb{Z}$-grading induced by $\operatorname{deg}\left(x_{i}\right)=\boldsymbol{a}_{i}>0$ and the ideal $B$ be $\left\langle x_{1}, \ldots, x_{n}\right\rangle$ as in Example 2.1. If $\mathcal{C}=\{\mathbf{1}\}$, then Remark 3.3 implies that $\operatorname{reg}(S)=\left\{\boldsymbol{m} \in \mathbb{Z}: \boldsymbol{m} \geq n-\boldsymbol{a}_{1}-\cdots-\boldsymbol{a}_{n}\right\}$. On the other hand, if $\boldsymbol{a}_{i}=1$ for $1 \leq i \leq n$ and $\mathcal{C}=\left\{\boldsymbol{c}_{1}, \ldots, \boldsymbol{c}_{\ell}\right\}$ with $0<\boldsymbol{c}_{1} \leq \cdots \leq \boldsymbol{c}_{\ell}$, then we have $\operatorname{reg}(S)=\left\{\boldsymbol{m} \in \mathbb{Z}: \boldsymbol{m} \geq(n-1)\left(\boldsymbol{c}_{\ell}-1\right)\right\}$.

Example 4.3. Let $t \in \mathbb{N}$. Suppose that $S$ is the homogeneous coordinate ring of the Hirzebruch surface $\mathbb{F}_{t}$; see Example 2.6. If $\mathcal{C}=\left\{\left[\begin{array}{l}1 \\ 0\end{array}\right],\left[\begin{array}{l}0 \\ 1\end{array}\right]\right\}$ then Example 3.5 implies that

$$
\operatorname{reg}(S)= \begin{cases}\mathbb{N}^{2} & \text { for } t=0,1 \\
\left(\left[\begin{array}{c}
t-1 \\
0
\end{array}\right]+\mathbb{N}^{2}\right) \cup\left(\left[\begin{array}{l}
0 \\
1
\end{array}\right]+\mathbb{N}^{2}\right) & \text { for } t \geq 2 .\end{cases}
$$

Observe that when $t \geq 2$ the set $\operatorname{reg}(S)$ is not determined by a single element of $G$. Moreover, we have int $\mathcal{K}^{\text {sat }} \subset \operatorname{reg}(S)$ for all $t \in \mathbb{N}$, but $\mathbf{0} \notin \operatorname{reg}(S)$ for $t \geq 2$. The shaded region in Figure 5 represents $\operatorname{reg}(S)$ when $t=2$.

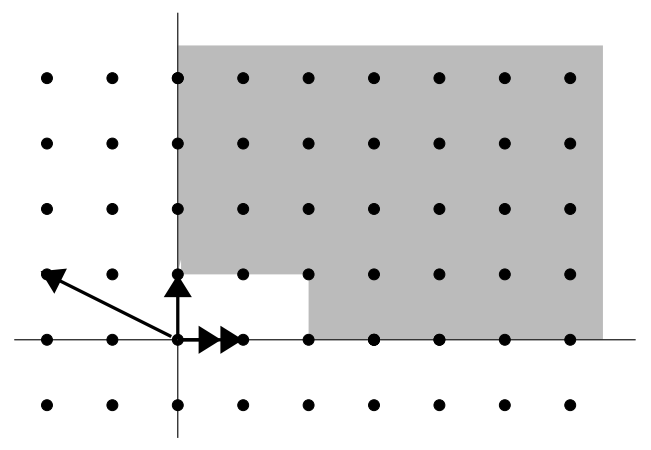

Figure 5. The $\operatorname{reg}(S)$ for the homogeneous coordinate ring $S$ of $\mathbb{F}_{2}$.

Without additional hypotheses, it is possible that $\operatorname{reg}(M)$ is the empty set. Indeed, if $S=\mathbb{k}\left[x_{1}, x_{2}, x_{3}\right]$ has the $\mathbb{Z}$-grading described in Example [3.10, then $\operatorname{reg}(S)=\emptyset$. Fortunately, there is a large class of pairs $(S, B)$ for which $\operatorname{reg}(M) \neq \emptyset$ for all finitely generated $S$-modules $M$. Specifically, we have the following:

Proposition 4.4. If $\operatorname{pos}(\overline{\mathcal{A}})$ is a pointed cone and $\mathbb{N} \mathcal{C} \cap$ int $\mathcal{K}^{\text {sat }} \neq \emptyset$, then every module $M$ is $\boldsymbol{m}$-regular for some $\boldsymbol{m} \in G$. 
Proof. Corollary 3.8 states that there is $\boldsymbol{m} \in \mathcal{K}^{\text {sat }}$ such that $H_{B}^{i}(M)_{\boldsymbol{p}}=0$ for all $i$ and all $\boldsymbol{p} \in \boldsymbol{m}+\mathcal{K}^{\text {sat }}$. By hypothesis, there exists $\boldsymbol{c} \in \mathbb{N} \mathcal{C} \cap \operatorname{int} \mathcal{K}^{\text {sat }}$. Remark 3.7 implies that there exists $k \in \mathbb{N}$ such that

$$
k \boldsymbol{c} \in \bigcap_{\substack{\lambda_{1}, \ldots, \lambda_{\ell} \in \mathbb{N} \\ \lambda_{1}+\cdots+\lambda_{\ell} \leq d}}\left(\boldsymbol{m}+\lambda_{1} \boldsymbol{c}_{1}+\cdots+\lambda_{\ell} \boldsymbol{c}_{\ell}+\mathcal{K}^{\mathrm{sat}}\right) .
$$

We conclude that $k c \in \operatorname{reg}(M)$.

To show that a module $M$ is $\boldsymbol{m}$-regular directly from Definition 4.1, one must verify that an infinite number of graded components of the local cohomology vanish. In fact, under the appropriate hypothesis, one need only check a finite number of components. To prove this result, we introduce the following two weaker versions of regularity.

Definition 4.5. Given $k \in \mathbb{N}$, the module $M$ is $\boldsymbol{m}$-regular from level $k$ if $H_{B}^{i}(M)_{\boldsymbol{p}}=0$ for all $i \geq k$ and all $\boldsymbol{p} \in \boldsymbol{m}+\mathbb{N} \boldsymbol{C}[1-i]$. In particular, $M$ is $\boldsymbol{m}$-regular if and only if it is $\boldsymbol{m}$-regular from level 0 . We set $\operatorname{reg}^{k}(M):=\{\boldsymbol{m} \in G: M$ is $\boldsymbol{m}$-regular from level $k\}$. For $k>0$, we say $M$ is weakly $\boldsymbol{m}$-regular from level $k$ if $H_{B}^{i}(M)_{\boldsymbol{p}}=0$ for all $i \geq k$ and all $\boldsymbol{p}=\boldsymbol{m}-\lambda_{1} \boldsymbol{c}_{1}-\cdots-\lambda_{\ell} \boldsymbol{c}_{\ell}$ where $\lambda_{j} \in \mathbb{N}$ and $\lambda_{1}+\cdots+\lambda_{\ell}=i-1$.

Our goal is to show that, when $\mathcal{C} \subseteq \mathcal{K}, M$ is $\boldsymbol{m}$-regular if and only if it is weakly $\boldsymbol{m}$-regular from level 1 and $H_{B}^{0}(M)_{\boldsymbol{p}}=0$ for $\boldsymbol{p} \in \bigcup_{1 \leq j \leq \ell}\left(\boldsymbol{m}+\boldsymbol{c}_{j}+\mathbb{N} \boldsymbol{e}\right)$. To accomplish this, we need the following fact.

Lemma 4.6. Let $k$ be a positive integer. If $M$ is weakly $\boldsymbol{m}$-regular from level $k$ and $g \in S_{\boldsymbol{c}_{j}}$ is almost a nonzerodivisor on $M$ for some $1 \leq j \leq \ell$, then $M / g_{j} M$ is also weakly $\boldsymbol{m}$-regular from level $k$.

Proof. Since $g_{j}$ is almost a nonzerodivisor on $M$, the submodule $\left(0:_{M} g_{j}\right)$ is a $B$-torsion module. We set $\bar{M}:=M /\left(0:_{M} g_{j}\right)$. Because $H_{B}^{i}\left(\left(0:_{M} g_{j}\right)\right)=0$ for $i>0$, the long exact sequence associated to the exact sequence $0 \longrightarrow\left(0:_{M} g_{j}\right) \longrightarrow M \longrightarrow \bar{M} \longrightarrow 0$ implies that $H_{B}^{i}(M)=H_{B}^{i}(\bar{M})$ for $i>0$. Since $M$ is weakly $\boldsymbol{m}$-regular from level $k$ and $k>0$, it follows that $\bar{M}$ is weakly $\boldsymbol{m}$-regular from level $k$. On the other hand, the long exact sequence associated to the exact sequence $0 \longrightarrow \bar{M}\left(-\boldsymbol{c}_{j}\right) \longrightarrow M \longrightarrow M / g_{j} M \longrightarrow 0$ contains

$$
H_{B}^{i}(M)_{\boldsymbol{p}} \longrightarrow H_{B}^{i}\left(M / g_{j} M\right)_{\boldsymbol{p}} \longrightarrow H_{B}^{i+1}(\bar{M})_{\boldsymbol{p}-\boldsymbol{c}_{j}} .
$$

Since $M$ and $\bar{M}$ are weakly $\boldsymbol{m}$-regular from level $k$, the left and right modules in (4.6.1) vanish when $i \geq k$ and $\boldsymbol{p}=\boldsymbol{m}-\lambda_{1} \boldsymbol{c}_{1}-\cdots-\lambda_{\ell} \boldsymbol{c}_{\ell}$ where $\lambda_{1}, \ldots, \lambda_{\ell} \in \mathbb{N}$ and $\lambda_{1}+\cdots+\lambda_{\ell}=i-1$. Therefore, the middle module also vanishes which proves that $M / g_{j} M$ is weakly $\boldsymbol{m}$-regular from level $k$.

Theorem 4.7. Let $k$ be a positive integer. If $\mathfrak{C} \subseteq \mathcal{K}$ and $M$ is weakly $\boldsymbol{m}$-regular from level $k$, then $M$ is weakly $\boldsymbol{p}$-regular from level $k$ for every $\boldsymbol{p} \in \boldsymbol{m}+\mathbb{N} \boldsymbol{C}$.

Proof. Since extension of our base field commutes with the formation of local cohomology, we may assume for the proof that $\mathbb{k}$ is infinite. Because $\mathcal{C} \subseteq \mathcal{K}$, Proposition 3.1 implies that we may choose an almost a nonzerodivisor $g_{j} \in S_{\boldsymbol{c}_{j}}$ on $M$ for each $1 \leq j \leq \ell$.

Suppose that $k>0$ and that $M$ is weakly $\boldsymbol{m}$-regular from level $k$. Since every $\boldsymbol{p} \in \boldsymbol{m}+\mathbb{N} \mathcal{C}$ can be expressed in the form $\boldsymbol{p}=\boldsymbol{m}+\lambda_{1} \boldsymbol{c}_{1}+\cdots+\lambda_{\ell} \boldsymbol{c}_{\ell}$ where $\lambda_{1}, \ldots, \lambda_{\ell} \in \mathbb{N}$, 
it suffices to the prove that if $M$ is weakly $\boldsymbol{q}$-regular from level $k$ then $M$ is also weakly $\left(\boldsymbol{q}+\boldsymbol{c}_{j}\right)$-regular from level $k$ for each $1 \leq j \leq \ell$. We proceed by induction on $\operatorname{dim} M$. If $\operatorname{dim} M=0$ then Grothendieck's vanishing theorem (Theorem 6.1.2 of [BrS]) implies that $H_{B}^{i}(M)=0$ for $i>0$. Thus, $M$ is weakly $\boldsymbol{q}$-regular from level $k$ for all $\boldsymbol{q} \in G$ and there is nothing more to prove.

Assume that $\operatorname{dim} M>0$ and set $\bar{M}:=M / H_{B}^{0}(M)$. Since $H_{B}^{0}(M)$ is a $B$-torsion module, the long exact sequence in cohomology arising from the short exact sequence $0 \longrightarrow H_{B}^{0}(M) \longrightarrow M \longrightarrow \bar{M} \longrightarrow 0$ implies that $H_{B}^{i}(M)=H_{B}^{i}(\bar{M})$ for all $i>0$. Hence, $\bar{M}$ is weakly $\boldsymbol{q}$-regular from level $k$ and it suffices to show that $\bar{M}$ is weakly $\left(\boldsymbol{q}+\boldsymbol{c}_{j}\right)$-regular from level $k$.

Since $g_{j}$ is a nonzerodivisor on $\bar{M}$, we have $\operatorname{dim} \bar{M} / g_{j} \bar{M}<\operatorname{dim} \bar{M}$. Lemma 4.6 shows that $\bar{M} / g_{j} \bar{M}$ is weakly $\boldsymbol{q}$-regular from level $k$ and the induction hypothesis implies $\bar{M} / g_{j} \bar{M}$ is also weakly $\left(\boldsymbol{q}+\boldsymbol{c}_{j}\right)$-regular from level $k$. Taking the long exact sequence associated to the exact sequence $0 \longrightarrow \bar{M}\left(-\boldsymbol{c}_{j}\right) \longrightarrow \bar{M} \longrightarrow \bar{M} / g_{j} \bar{M} \longrightarrow 0$, we obtain the exact sequence $H_{B}^{i}(\bar{M})_{\boldsymbol{q}^{\prime}} \longrightarrow H_{B}^{i}(\bar{M})_{\boldsymbol{q}^{\prime}+\boldsymbol{c}_{j}} \longrightarrow H_{B}^{i}\left(\bar{M} / g_{j} \bar{M}\right)_{\boldsymbol{q}^{\prime}+\boldsymbol{c}_{j}}$. Since $\bar{M}$ is weakly $\boldsymbol{q}$-regular from level $k$ and $\bar{M} / g_{j} \bar{M}$ is $\left(\boldsymbol{q}+\boldsymbol{c}_{j}\right)$-regular from level $k$, the left and right modules vanishes when $i \geq k$ and $\boldsymbol{q}^{\prime}=\boldsymbol{q}-\lambda_{1} \boldsymbol{c}_{1}-\cdots-\lambda_{\ell} \boldsymbol{c}_{\ell}$ where $\lambda_{1}, \ldots, \lambda_{\ell} \in \mathbb{N}$ and $\lambda_{1}+\cdots+\lambda_{\ell}=i-1$. Therefore, the middle module also vanishes which proves $\bar{M}$ is weakly $\left(\boldsymbol{q}+\boldsymbol{c}_{j}\right)$-regular from level $k$.

Theorem 4.7 provides the desired alternative characterization of $\boldsymbol{m}$-regularity.

Corollary 4.8. Let $\mathfrak{C} \subseteq \mathcal{K}$. The module $M$ is $\boldsymbol{m}$-regular if and only if $M$ is weakly $\boldsymbol{m}$-regular from level 1 and $H_{B}^{0}(M)_{\boldsymbol{p}}=0$ for all $\boldsymbol{p} \in \boldsymbol{m}+\left(\bigcup_{1 \leq j \leq \ell}\left(\boldsymbol{c}_{j}+\mathbb{N} \boldsymbol{C}\right)\right)$.

Proof. Suppose that the module $M$ is $\boldsymbol{m}$-regular from level 1 and $H_{B}^{0}(M)_{\boldsymbol{p}}$ vanishes for all $\boldsymbol{p} \in \boldsymbol{m}+\mathbb{N} \mathcal{C}[1]$. Since the condition on $H_{B}^{0}(M)_{\boldsymbol{p}}$ is the same as in Definition 4.1. we only need to show that $H_{B}^{i}(M)_{\boldsymbol{p}}=0$ for all $\boldsymbol{p} \in \boldsymbol{m}+\mathbb{N} \mathcal{e}[1-i]$. However, this is the content of Theorem 4.7. The converse follows directly from the Definition 4.1 and Definition 4.5.

The next example illustrates that the condition $\mathcal{C} \subseteq \mathcal{K}$ is necessary for Theorem 4.7

Example 4.9. Let $S=\mathbb{k}\left[x_{1}, x_{2}\right]$ have the $\mathbb{Z}$-grading defined by $\operatorname{deg}\left(x_{1}\right)=\mathbf{2}$ and $\operatorname{deg}\left(x_{2}\right)=3$ and let $B=\left\langle x_{1}, x_{2}\right\rangle$. Example 2.1 shows that $\mathcal{K}=6 \mathbb{N}$. Proposition 3.2 establishes that the nonvanishing local cohomology is concentrated in $H_{B}^{2}(S)$ and that $H_{B}^{2}(S)$ in nonzero exactly in degrees $\mathbf{- 5}, \mathbf{- 7}, \mathbf{- 8}, \mathbf{- 9}, \ldots$ If $\boldsymbol{e}=\{\mathbf{1}\} \not \subset \mathcal{K}$, then $S$ is weakly $(-5)$-regular from level 1 but not weakly $(-4)$-regular from level 1 . The strategy used in the proof of Theorem 4.7 does not apply because $S_{\mathbf{1}}=\emptyset$, so there is no nonzerodivisor of degree $\mathbf{1}$.

By design, the regularity of a module measures where its cohomological complexities vanish. Since Gröbner bases calculations are linked to homological properties, one expects a strong connection between regularity and computational complexity. Both Proposition 6.13 and Corollary 7.3 provide further support for this idea by relating regularity to initial modules and free resolutions. To make the connection between regularity and complexity more precise, we are interested in the following open problem:

Problem 4.10. Give a computationally efficient method of calculating $\operatorname{reg}(M)$. 
When $S$ has the standard grading, this problem is solved in $\mathrm{BmS}$ by showing that $\operatorname{reg}(M)$ is determined by the largest degree generator of the initial module $\operatorname{in}(M)$ with respect to a reverse lexicographic order in generic coordinates. Unfortunately, this technique does not extend directly polynomial rings with arbitrary multigradings. As the next example demonstrates, there may not be any coordinate changes which preserve the grading and change the initial module.

Example 4.11. Let $S$ be the homogeneous coordinate ring of a toric variety $X$ obtained from $\mathbb{P}^{2}$ by a sequence of five blow-ups. More explicitly, the minimal lattice points $\mathcal{B}$ on the rays of the fan corresponds to the columns of the matrix $\left[\begin{array}{llllllll}1 & 1 & 0 & -1 & -1 & -1 & 0 & 1 \\ 0 & 1 & 1 & 1 & 0 & -1 & -1 & -1\end{array}\right]$ and the associated irrelevant ideal is

$$
\begin{aligned}
B= & \left\langle x_{3} x_{4} x_{5} x_{6} x_{7} x_{8}, x_{1} x_{4} x_{5} x_{6} x_{7} x_{8}, x_{1} x_{2} x_{5} x_{6} x_{7} x_{8}, x_{1} x_{2} x_{3} x_{6} x_{7} x_{8},\right. \\
& \left.x_{1} x_{2} x_{3} x_{4} x_{7} x_{8}, x_{1} x_{2} x_{3} x_{4} x_{5} x_{8}, x_{2} x_{3} x_{4} x_{5} x_{6} x_{7}, x_{1} x_{2} x_{3} x_{4} x_{5} x_{6}\right\rangle .
\end{aligned}
$$

Hence, $G=\mathbb{Z}^{6}$ and we may assume that $\mathcal{A}$ be given by the columns of the matrix

$$
\left[\begin{array}{cccccccc}
1 & 0 & 0 & 0 & 0 & 0 & 1 & -1 \\
0 & 1 & -2 & 1 & 0 & 0 & 0 & 0 \\
0 & 0 & 1 & -1 & 1 & 0 & 0 & 0 \\
0 & 0 & 0 & 1 & -2 & 1 & 0 & 0 \\
0 & 0 & 0 & 0 & 1 & -1 & 1 & 0 \\
0 & 0 & 0 & 0 & 0 & 1 & -2 & 1
\end{array}\right] .
$$

The homogeneous coordinate ring $S=\mathbb{k}\left[x_{1}, \ldots, x_{8}\right]$ has the $\mathbb{Z}^{6}$-grading induced by $\operatorname{deg}\left(x_{i}\right)=\boldsymbol{a}_{i}$ for $1 \leq i \leq 8$. Since $S_{\boldsymbol{a}_{i}}$ is the $\mathbb{k}$-span of the variable $x_{i}$ for $1 \leq i \leq 8$, Corollary 4.7 in $\mathrm{Co} 2$ establishes that $\operatorname{Aut}(S)=\left(\mathbb{k}^{*}\right)^{8}$. As a consequence, any change of coordinates which preserves the grading does not alter the initial ideal. Hence, one cannot develop a theory of generic initial ideals.

\section{Degrees of Generators}

The regularity of a module should be regarded as a measure of its complexity. In this section, we justify this idea by proving that the regularity controls the degrees of the minimal generators. To understand the minimal generators of a module, we study submodules of the following form.

Definition 5.1. Let $\mathcal{D}$ be a subset of $G$. We define the $\mathcal{D}$-truncation of $M$, denoted $\left.M\right|_{\mathcal{D}}$, to be the submodule of $M$ generated by all the homogeneous elements in $M$ of degree $\boldsymbol{p}$ where $\boldsymbol{p} \in \mathcal{D}$.

In contrast with the standard graded case, the next example illustrates that $\left(\left.M\right|_{\mathcal{D}}\right)_{\boldsymbol{p}}$ may be nonzero even if $\boldsymbol{p} \notin \mathcal{D}$.

Example 5.2. Suppose that $S$ is the homogeneous coordinate ring of the Hirzebruch surface $\mathbb{F}_{2}$; see Examples 2.2 \& 2.6. If $\mathcal{D}:=\left[\begin{array}{l}1 \\ 1\end{array}\right]+\mathbb{N}^{2}$, then $\left.S\right|_{\mathcal{D}}$ is generated in degree $\left[\begin{array}{l}1 \\ 1\end{array}\right]$. Observe that $\left.x_{1}^{3} x_{2}^{2} \in S\right|_{\mathcal{D}}$ but $\operatorname{deg}\left(x_{1}^{3} x_{2}^{2}\right) \notin \mathcal{D}$.

To prove the main theorem in this section, we need the following fact.

Lemma 5.3. Let $\boldsymbol{c} \in \mathbb{N} \mathcal{C}$ and let $\mathcal{V} \subseteq G$ such that $H_{B}^{0}(M)_{\boldsymbol{p}}=0$ for all $\boldsymbol{p} \in \mathcal{V}$. If $M$ is $\boldsymbol{m}$-regular from level 1 and $g \in S_{\boldsymbol{c}}$ is almost a nonzerodivisor on $M$, then $H_{B}^{0}(M / g M)_{\boldsymbol{p}}=0$ for all $\boldsymbol{p} \in \mathcal{V} \cap(\boldsymbol{m}+\boldsymbol{c}+\mathbb{N} \mathcal{C})$. 
Proof. Set $\bar{M}:=M /\left(0:_{M} g\right)$. Because $g$ is almost a nonzerodivisor on $M,\left(0:_{M} g\right)$ is a $B$-torsion module and the long exact sequence associated to the short exact sequence $0 \longrightarrow\left(0:_{M} g\right) \longrightarrow M \longrightarrow \bar{M} \longrightarrow 0$ implies that $H_{B}^{i}(M)=H_{B}^{i}(\bar{M})$ for $i>0$. Since $M$ is $\boldsymbol{m}$-regular from level 1 , it follows that $\bar{M}$ is also $\boldsymbol{m}$-regular from level 1 . Now, the long exact sequence associated to the exact sequence $0 \longrightarrow \bar{M}(-\boldsymbol{c}) \longrightarrow M \longrightarrow M / g M \longrightarrow 0$ contains $H_{B}^{0}(M)_{\boldsymbol{p}} \longrightarrow H_{B}^{0}(M / g M)_{\boldsymbol{p}} \longrightarrow H_{B}^{1}(\bar{M})_{\boldsymbol{p}-\boldsymbol{c}}$. By hypothesis, the left module vanishes when $\boldsymbol{p} \in \mathcal{V}$. Since $\bar{M}$ is $\boldsymbol{m}$-regular from level 1 , the right module vanishes for all $\boldsymbol{p} \in \boldsymbol{m}+\boldsymbol{c}+\mathbb{N} \mathcal{C}$. Hence, the middle module vanishes for all $\boldsymbol{p} \in \mathcal{V} \cap(\boldsymbol{m}+\boldsymbol{c}+\mathbb{N} \boldsymbol{C})$.

We now prove that if $M$ is $\boldsymbol{m}$-regular then $\left.M\right|_{\boldsymbol{m}}=\left.M\right|_{(\boldsymbol{m}+\mathbb{N} \mathfrak{C})}$. In the standard graded case, this is true for any $\boldsymbol{m} \in \mathbb{Z}$ that is larger than the maximum degree of the minimal generators.

Theorem 5.4. Assume that $\mathfrak{C} \subseteq \mathcal{K}$. If the module $M$ is $\boldsymbol{m}$-regular, then we have $\left.M\right|_{\boldsymbol{m}}=\left.M\right|_{(\boldsymbol{m}+\mathbb{N} \mathfrak{C})}$. In particular, the degrees of the minimal generators of $M$ do not belong to the set $\operatorname{reg}(M)+\left(\bigcup_{1 \leq j \leq \ell}\left(\boldsymbol{c}_{j}+\mathbb{N} \boldsymbol{C}\right)\right)$.

Proof. We prove the following claim: If $M$ is $\boldsymbol{m}$-regular from level 1 and $H_{B}^{0}(M)_{\boldsymbol{p}}=0$ for all $\boldsymbol{p} \in \mathcal{V}$, then $\left.M\right|_{(\boldsymbol{m}+\mathbb{N} \boldsymbol{C}) \cap \mathcal{V}}$ is a submodule of $\left.M\right|_{\boldsymbol{m}}$. We proceed by induction on $\operatorname{dim} M$. If $\operatorname{dim} M<0$, which is equivalent to saying that $M=0$, then $\left.M\right|_{(\boldsymbol{m}+\mathbb{N} \mathfrak{e}) \cap \mathcal{v}}=0$ is trivially a submodule of $M_{\boldsymbol{m}}$.

Suppose that $\operatorname{dim} M \geq 0$. Set $\bar{M}:=M / H_{B}^{0}(M)$ and consider the short exact sequence

$$
0 \longrightarrow H_{B}^{0}(M) \longrightarrow M \longrightarrow \bar{M} \longrightarrow 0 \text {. }
$$

We claim that it is enough to prove that $\left.\bar{M}\right|_{(\boldsymbol{m}+\mathbb{N} \boldsymbol{e})}$ is a submodule of $\left.\bar{M}\right|_{\boldsymbol{m}}$. To see this, let $\boldsymbol{p} \in(\boldsymbol{m}+\mathbb{N} \mathcal{C}) \cap \mathcal{V}$ and let $f \in M_{\boldsymbol{p}}$. If $\bar{f} \in \bar{M}_{\boldsymbol{p}}$ can be written as $\sum_{i} s_{i} \bar{f}_{i}$ for some $s_{i} \in S$ and some $\bar{f}_{i} \in \bar{M}_{\boldsymbol{m}}$, then $f=\sum_{i} s_{i} f_{i}+h$ for $h \in H_{B}^{0}(M)_{\boldsymbol{p}}$. Since $\boldsymbol{p} \in \mathcal{V}$, we have $H_{B}^{0}(M)_{\boldsymbol{p}}=0$ which implies $h=0$. Thus, $f=\sum_{i} s_{i} f_{i}$ and it suffices to prove the claim for $\bar{M}$. The long exact sequence associated to (5.4.1) implies $H_{B}^{i}(M)=H_{B}^{i}(\bar{M})$ for all $i>0$. Since $M$ is $\boldsymbol{m}$-regular from level 1 and $H_{B}^{0}(\bar{M})=0$, we deduce that $\bar{M}$ is $\boldsymbol{m}$-regular.

Extending the base field commutes with the computing local cohomology, so we may assume without loss of generality that $\mathbb{k}$ is infinite. Because $\mathcal{C} \subseteq \mathcal{K}$, Proposition 3.1 implies that, for each $1 \leq j \leq \ell$, we may choose a nonzerodivisor $g_{j} \in S_{\boldsymbol{c}_{j}}$ on $\bar{M}$. It follows from Lemma 4.6 that $\bar{M} / g_{j} \bar{M}$ is $\boldsymbol{m}$-regular from level 1 and Lemma 5.3 shows that $H_{B}^{0}\left(\bar{M} / g_{j} \bar{M}\right)_{\boldsymbol{p}}=0$ for all $\boldsymbol{p} \in \boldsymbol{m}+\boldsymbol{c}_{j}+\mathbb{N} \boldsymbol{C}$. Since $\operatorname{dim} \bar{M} / g_{j} \bar{M}<\operatorname{dim} \bar{M} \leq \operatorname{dim} M$, the induction hypothesis with $\mathcal{V}=\boldsymbol{m}+\boldsymbol{c}_{j}+\mathbb{N} \mathcal{C}$ guarantees that $\left.\left(\bar{M} / g_{j} \bar{M}\right)\right|_{\left(\boldsymbol{m}+\boldsymbol{c}_{j}+\mathbb{N} \boldsymbol{e}\right)}$ is a submodule of $\left.\left(\bar{M} / g_{j} \bar{M}\right)\right|_{\boldsymbol{m}}$. It remains to show that this implies $\left.\bar{M}\right|_{(\boldsymbol{m}+\mathbb{N} \boldsymbol{e})}$ is a submodule of $\left.\bar{M}\right|_{\boldsymbol{m}}$.

Suppose otherwise. For each element $f \in \bar{M}$ with $\operatorname{deg}(f) \in \boldsymbol{m}+\mathbb{N} \boldsymbol{C}$, we set $\operatorname{reach}(f):=\min \left\{\lambda_{1}+\cdots+\lambda_{\ell}: \operatorname{deg}(f)=\boldsymbol{m}+\lambda_{1} \boldsymbol{c}_{1}+\cdots+\lambda_{\ell} \boldsymbol{c}_{\ell}\right.$ and $\left.\lambda_{1}, \ldots, \lambda_{\ell} \in \mathbb{N}\right\}$.

Because $\bar{M}$ is noetherian and we are assuming that $\left.\left.\bar{M}\right|_{(\boldsymbol{m}+\mathbb{N} \mathcal{e})} \nsubseteq \bar{M}\right|_{\boldsymbol{m}}$, there is a minimal generator $\left.f \in \bar{M}\right|_{(\boldsymbol{m}+\mathbb{N} \mathfrak{e})}$ which has smallest reach $(f)$ among all the minimal generators of $\left.\bar{M}\right|_{(\boldsymbol{m}+\mathbb{N} \mathfrak{C})}$ with degree not equal to $\boldsymbol{m}$. Since $\left.f \notin \bar{M}\right|_{\boldsymbol{m}}$, we have $\operatorname{reach}(f)>0$ and 
there exists $\boldsymbol{c}_{j}$ with $1 \leq j \leq \ell$ such that $\operatorname{deg}(f) \in \boldsymbol{m}+\boldsymbol{c}_{j}+\mathbb{N} \mathcal{C}$. From the previous paragraph, we know that the image of $f$ in $\left.\left(\bar{M} / g_{j} \bar{M}\right)\right|_{\left(\boldsymbol{m}+\boldsymbol{c}_{j}+\mathbb{N} \boldsymbol{e}\right)}$ belongs to $\left.\left(\bar{M} / g_{j} \bar{M}\right)\right|_{\boldsymbol{m}}$. Hence, we may choose homogeneous elements $f_{1}, \ldots,\left.f_{e} \in \bar{M}\right|_{\boldsymbol{m}}$ and $s_{1}, \ldots, s_{e} \in S$ such that $f-s_{1} f_{1}-\cdots-\left.s_{e} f_{e} \in\left(g_{j} \bar{M}\right)\right|_{\left(\boldsymbol{m}+\boldsymbol{c}_{j}+\mathbb{N} \mathfrak{e}\right)} \subseteq g_{j}\left(\left.\bar{M}\right|_{(\boldsymbol{m}+\mathbb{N} \mathfrak{e})}\right)$. Let $f^{\prime}$ be the homogeneous element of $\left.\bar{M}\right|_{(\boldsymbol{m}+\mathbb{N} \mathcal{C})}$ satisfying the equation $f-s_{1} f_{1}-\cdots-s_{e} f_{e}=g_{j} f^{\prime}$. Since $f$ does not belong to $\left.\bar{M}\right|_{\boldsymbol{m}}$, the element $f^{\prime}$ cannot belong to $\left.\bar{M}\right|_{\boldsymbol{m}}$. However, reach $\left(f^{\prime}\right)<\operatorname{reach}(f)$ and $\left.f^{\prime} \in \bar{M}\right|_{(\boldsymbol{m}+\mathbb{N} \mathcal{C})}$ which contracts the our choice of $f$. We conclude that $\left.\bar{M}\right|_{(\boldsymbol{m}+\mathbb{N} \mathcal{C})}$ is a submodule of $\bar{M}_{\boldsymbol{m}}$.

Proof of Theorem 1.3, In the introduction we assumed that $\mathcal{C}$ was the minimal generating set of $\mathcal{K}$. Hence, Theorem 1.3 follows from Theorem 5.4 .

When $S$ has the standard grading and $\mathcal{C}=\{\mathbf{1}\}$, the statement that the minimal generators of $M$ do not lie in $\boldsymbol{m}+\mathbb{N} \mathfrak{e}[1]$ is equivalent to saying that the minimal generators of $M$ have degree at most $\boldsymbol{m}$. In this case, Theorem 5.4 proves that the regularity gives a bound on the degrees of the minimal generators. For an ideal in $S$, an upper bound on the minimal generators yields a finite set containing the degrees of the minimal generators. In contrast, Theorem 5.4 does not automatically produce a finite set containing the degrees of the minimal generators for an ideal when $S$ has a general multigrading.

We may still ask the question whether if $M$ is $\boldsymbol{m}$-regular all minimal generators of $M$ lie in $\boldsymbol{m}-\mathbb{N} \mathcal{C}$. It is not hard to find examples showing that this need not be the case, but to date all such examples have had $H_{B}^{0}(M) \neq 0$. In the bigraded case [HW] take a different approach, adding an extra local cohomology vanishing requirement to guarantee this bound on generators.

\section{Regularity of $\mathscr{O}_{X}$-MOdules}

In this section, we develop a multigraded version of regularity for coherent sheaves on a simplicial toric variety $X$. Since $\mathscr{O}_{X}$-modules correspond to finitely generated modules over the homogeneous coordinate ring of $X$, regularity for $\mathscr{O}_{X}$-modules is essentially a special case of regularity for $S$-modules. Nevertheless, the geometric context provides new interpretations and applications for regularity.

To begin, we examine the relation between sheaves on a toric variety $X$ and $G$-graded modules over its homogeneous coordinate ring $S$; see [Co2]. For $\sigma \in \Delta$, we write $\boldsymbol{x}_{\widehat{\sigma}}$ for the monomial $\prod_{i \notin \sigma} x_{i} \in S$ and $U_{\sigma} \cong \operatorname{Spec}\left(S\left[\boldsymbol{x}_{\widehat{\sigma}}^{-1}\right]_{\mathbf{0}}\right)$ is the corresponding open affine subset of $X$. Every (not necessarily finitely generated) $G$-graded $S$-module $F$ gives rise to a quasicoherent sheaf $\widetilde{F}$ on $X$ where $H^{0}\left(U_{\sigma}, \widetilde{F}\right)=\left(F\left[\boldsymbol{x}_{\widehat{\sigma}}^{-1}\right]\right)_{\mathbf{0}}$. If $M$ is finitely generated $G$-graded $S$-module, then $\widetilde{M}$ is a coherent $\mathscr{O}_{X}$-module. Moreover, every quasicoherent sheaf on $X$ is of this form for some $G$-graded $S$-module $F$ and if the sheaf is coherent then $F$ can be taken to be finitely generated. For $\boldsymbol{p} \in G$, the sheaf associated to the module $S(\boldsymbol{p})$ is denoted $\mathscr{O}_{X}(\boldsymbol{p})$. Note that the natural map $\mathscr{O}_{X}(\boldsymbol{p}) \otimes_{\mathscr{O}_{X}} \mathscr{O}_{X}(\boldsymbol{q}) \longrightarrow \mathscr{O}_{X}(\boldsymbol{p}+\boldsymbol{q})$ need not be an isomorphism. For an $\mathscr{O}_{X}$-module $\mathscr{F}$, we simply write $\mathscr{F}(\boldsymbol{p})$ for $\mathscr{F} \otimes_{\mathscr{O}_{X}} \mathscr{O}_{X}(\boldsymbol{p})$. 
The map sending a finitely generated $G$-graded $S$-module $M$ to the sheaf $\widetilde{M}$ is not injective. In fact, there are many nonzero modules which give the zero sheaf. The following proposition analyzes this phenomenon.

Proposition 6.1. The sheaf $\widetilde{M}$ is zero if and only if there is $j>0$ such that $B^{j} M_{\boldsymbol{p}}=0$ for all $\boldsymbol{p} \in \mathcal{K}$.

Proof. Suppose that $B^{j} M_{\boldsymbol{p}}=0$ for all $\boldsymbol{p} \in \mathcal{K}$. Fix $\sigma \in \Delta$ and take $f / \boldsymbol{x}^{\boldsymbol{u}} \in\left(M\left[\boldsymbol{x}_{\widehat{\sigma}}^{-1}\right]\right)_{\mathbf{0}}$. Remark 3.7 implies that there exists a monomial $\boldsymbol{x}^{\boldsymbol{v}} \in S$ such that $\operatorname{supp}(\boldsymbol{v}) \subseteq \widehat{\sigma}$ and $\operatorname{deg}\left(\boldsymbol{x}^{\boldsymbol{u}+\boldsymbol{v}}\right) \in \mathcal{K}^{\text {sat }}$. Moreover, we may choose $k \in \mathbb{N}$ such that $\boldsymbol{p}:=\operatorname{deg}\left(\boldsymbol{x}^{k \boldsymbol{u}+k \boldsymbol{v}}\right) \in \mathcal{K}$. It follows that $\boldsymbol{x}^{(k-1) \boldsymbol{u}+k \boldsymbol{v}} f \in M_{\boldsymbol{p}}$ and $\left(\boldsymbol{x}_{\widehat{\sigma}}\right)^{j} \boldsymbol{x}^{(k-1) \boldsymbol{u}+k \boldsymbol{v}} f=0$ for some $j>0$. Therefore, $f / \boldsymbol{x}^{\boldsymbol{u}}=0$ and $\left(M\left[\boldsymbol{x}_{\widehat{\sigma}}^{-1}\right]\right)_{\mathbf{0}}=0$ which shows that $\widetilde{M}$ is the zero sheaf.

Conversely, suppose that $\widetilde{M}$ is the zero sheaf, fix $\boldsymbol{p} \in \mathcal{K}$ and let $f \in M_{\boldsymbol{p}}$. Lemma 2.4 implies that for each $\sigma \in \Delta$ there is a monomial $\boldsymbol{x}^{\boldsymbol{u}} \in S \operatorname{such}$ that $\operatorname{deg}\left(\boldsymbol{x}^{\boldsymbol{u}}\right)=\boldsymbol{p}$ and $\operatorname{supp}(\boldsymbol{u}) \subseteq \widehat{\sigma}$. The monomial $\boldsymbol{x}^{\boldsymbol{u}}$ is invertible in $S\left[\boldsymbol{x}_{\widehat{\sigma}}^{-1}\right]$ and $f / \boldsymbol{x}^{\boldsymbol{u}} \in\left(M\left[\boldsymbol{x}_{\widehat{\sigma}}^{-1}\right]\right)_{\mathbf{0}}=0$. It follows that $\left(\boldsymbol{x}_{\widehat{\sigma}}\right)^{j} f=0$ for some $j>0$. To see that one $j$ works for all $f \in M_{\boldsymbol{p}}$ and all $\boldsymbol{p} \in \mathcal{K}$, observe that $\left.M\right|_{\mathcal{K}}$ is a submodule of $M$ and thus finitely generated. The proposition now follows easily.

As above, we continue to write $\mathcal{C}=\left\{\boldsymbol{c}_{1}, \ldots, \boldsymbol{c}_{\ell}\right\}$ for a fixed subset of $G$. Geometrically, the set $\mathcal{C}$ corresponds to choosing a collection of reflexive sheaves on $X$. In particular, fixing an ample line bundle on $X$ determines a set $\mathcal{C}$ consisting of one element. At the other extreme, the toric variety $X$ is equipped with a canonical choice for $\mathcal{C}$, namely the minimal generators of the semigroup $\mathcal{K}$. We are most interested in this case. Nonetheless, we expect that the flexibility in allowing other sets $\mathcal{C}$ will also be useful in studying how maps between toric varieties effect regularity.

In analogy with Definition 4.1, we have the following:

Definition 6.2. If $\boldsymbol{m} \in G$, then an $\mathscr{O}_{X}$-module $\mathscr{F}$ is $\boldsymbol{m}$-regular (with respect to $\mathcal{C}$ ) if $H^{i}(X, \mathscr{F}(\boldsymbol{p}))=0$ for all $i>0$ and all $\boldsymbol{p} \in \boldsymbol{m}+\mathbb{N} \mathfrak{C}[-i]$. We write $\operatorname{reg}(\mathscr{F})$ for the set $\{\boldsymbol{m} \in G: \mathscr{F}$ is $\boldsymbol{m}$-regular $\}$.

Remark 6.3. Notice that if $\mathscr{F}$ is $\boldsymbol{m}$-regular then $\mathscr{F}$ is $\boldsymbol{p}$-regular for all $\boldsymbol{p} \in \boldsymbol{m}+\mathbb{N} \mathcal{C}$.

To make an explicit connection between the two definitions of regularity, we first recall the following. If $\mathscr{F}$ is the coherent $\mathscr{O}_{X}$-module corresponding to $M$, then the local cohomology is related to the (Zariski) cohomology of sheaves by the exact sequence

$$
0 \longrightarrow H_{B}^{0}(M) \longrightarrow M \longrightarrow \bigoplus_{\boldsymbol{p} \in G} H^{0}(X, \mathscr{F}(\boldsymbol{p})) \longrightarrow H_{B}^{1}(M) \longrightarrow 0
$$

and $H_{B}^{i+1}(M) \cong \bigoplus_{\boldsymbol{p} \in G} H^{i}(X, \mathscr{F}(\boldsymbol{p}))$ for all $i \geq 1$; see Proposition 2.3 in [EMS]. Using this observation, we have:

Proposition 6.4. Let $\mathscr{F}$ be the coherent $\mathscr{O}_{X}$-module associated to the module $M$. The sheaf $\mathscr{F}$ is $\boldsymbol{m}$-regular if and only if $M$ is $\boldsymbol{m}$-regular from level 2 . The module $M$ is $\boldsymbol{m}$-regular if and only if the following three conditions hold:

1. $\mathscr{F}$ is $\boldsymbol{m}$-regular;

2. the natural map $M_{\boldsymbol{p}} \longrightarrow H^{0}(X, \mathscr{F}(\boldsymbol{p}))$ is surjective for all $\boldsymbol{p} \in \boldsymbol{m}+\mathbb{N} \boldsymbol{C}$; and

3. $H_{B}^{0}(M)_{\boldsymbol{p}}=0$ for all $\boldsymbol{p} \in \bigcup_{1 \leq j \leq \ell}\left(\boldsymbol{m}+\boldsymbol{c}_{j}+\mathbb{N} \boldsymbol{C}\right)$. 
Proof. Since $H_{B}^{i+1}(M)_{\boldsymbol{p}} \cong H^{i}(X, \mathscr{F}(\boldsymbol{p}))$ for all $i \geq 1$, we see that $\mathscr{F}$ is $\boldsymbol{m}$-regular if and only if $M$ is $\boldsymbol{m}$-regular from level 2 . To establish the second part, observe that saying $M$ is $\boldsymbol{m}$-regular is equivalent to saying that $M$ is $\boldsymbol{m}$-regular from level $2, H_{B}^{1}(M)_{\boldsymbol{p}}=0$ for all $\boldsymbol{p} \in \boldsymbol{m}+\mathbb{N} \boldsymbol{C}$ and $H_{B}^{0}(M)_{\boldsymbol{p}}=0$ for all $\boldsymbol{p} \in \boldsymbol{m}+\bigcup_{1 \leq j \leq \ell}\left(\boldsymbol{c}_{j}+\mathbb{N} \boldsymbol{e}\right)$. Since the exact sequence (6.3.1) implies that $H_{B}^{1}(M)_{\boldsymbol{p}}=0$ if and only if $M_{\boldsymbol{p}} \longrightarrow H^{0}(X, \mathscr{F}(\boldsymbol{p}))$ is surjective, the assertion follows.

Example 6.5. Since $H_{B}^{i}(S)=0$ for $i=0,1$ (see Remark 3.3), Proposition 6.4 implies that $\operatorname{reg}\left(\mathscr{O}_{X}\right)=\operatorname{reg}(S)$. In particular, Example 4.2 shows that $\operatorname{reg}\left(\mathscr{O}_{\mathbb{P}^{d}}\right)=\mathbb{N}$ when $\mathcal{C}=\{1\}$.

The next corollary shows that Definition 6.2 extends the original definition given in $\S 14$ in Mum. When $X=\mathbb{P}^{d}$ and $\mathcal{C}=\{\mathbf{1}\}\left(\mathbf{1} \in G\right.$ corresponds to $\left.\mathscr{O}_{X}(\mathbf{1}) \in \operatorname{Pic}\left(\mathbb{P}^{d}\right)\right)$, the second part of this corollary is Mumford's definition.

Corollary 6.6. Let $\mathscr{F}$ be the coherent $\mathscr{O}_{X}$-module associated to the module $M$. If $\mathcal{C} \subseteq \mathcal{K}$ then the following are equivalent:

1. $\mathscr{F}$ is $\boldsymbol{m}$-regular;

2. $H^{i}\left(X, \mathscr{F}\left(\boldsymbol{m}-\lambda_{1} \boldsymbol{c}_{1}-\cdots-\lambda_{\ell} \boldsymbol{c}_{\ell}\right)\right)=0$ for all $i>0$ and all $\lambda_{1}, \ldots, \lambda_{\ell} \in \mathbb{N}$ satisfying $\lambda_{1}+\cdots+\lambda_{\ell}=i$.

Proof. This follows from Proposition 6.4 and Corollary 4.8.

The regularity of a finite set of points has a geometric interpretation. Example 2.16 in MS rephrases the following result in terms of the associated multigraded Hilbert polynomial.

Proposition 6.7. Assume that $\mathfrak{C} \subseteq \mathcal{K}$. Let $Y$ be an artinian subscheme of $X$ of length $t$ (for example, a set of $t$ points in $X$ ) and let $I_{Y}$ be the associated $B$-saturated ideal in $S$. If $R_{Y}=S / I_{Y}$ then $\boldsymbol{m} \in \operatorname{reg}\left(R_{Y}\right)$ if and only if the space of forms vanishing on $Y$ has codimension $t$ is the space of forms of degree $\boldsymbol{m}$.

Proof. Since $I_{Y}$ is $B$-saturated, the local cohomology module $H_{B}^{0}\left(R_{Y}\right)$ vanishes. Because $\operatorname{dim} Y=0$, we also have $H_{B}^{i+1}\left(R_{Y}\right)=\bigoplus_{\boldsymbol{p} \in G} H^{i}\left(X, \mathscr{O}_{Y}(\boldsymbol{p})\right)=0$ for all $i>0$. Hence, $\operatorname{reg}\left(R_{Y}\right)$ is determined by the module $H_{B}^{1}\left(R_{Y}\right)$. Since the exact sequence (6.3.1) becomes $0 \longrightarrow R_{Y} \longrightarrow \bigoplus_{\boldsymbol{p} \in G} H^{0}\left(X, \mathscr{O}_{Y}(\boldsymbol{p})\right) \longrightarrow H_{B}^{1}\left(R_{Y}\right) \longrightarrow 0$, we see that $\boldsymbol{m} \in \operatorname{reg}\left(R_{Y}\right)$ if and only if $\left(R_{Y}\right)_{\boldsymbol{m}} \longrightarrow H^{0}\left(X, \mathscr{O}_{Y}(\boldsymbol{m})\right)$ is surjective. Again, because $\operatorname{dim} Y=0$, the scheme $Y$ is an affine variety and every reflexive sheaf on $Y$ is trivial. It follows that for all $\boldsymbol{p}$ we have $\mathscr{O}_{Y}(\boldsymbol{p}) \cong \mathscr{O}_{Y}$ and $\operatorname{dim}_{\mathbb{k}} H^{0}\left(X, \mathscr{O}_{Y}\right)=t$. Therefore, $R_{Y}$ is $\boldsymbol{m}$-regular if and only if $\operatorname{dim}_{\mathbb{k}}\left(R_{Y}\right)_{\boldsymbol{m}}=t$. In other words, the space of forms $\left(I_{Y}\right)_{\boldsymbol{m}}$ that vanish on $Y$ has codimension $t$.

Although the definition of regularity may seem rather technical ( "apparently silly" in Mumford's words), the following theorem provides a geometric interpretation for regularity. Concretely, the regularity of a coherent sheaf measures how much one has to twist for the sheaf to be generated by its global sections. We first show that the module $M$ and certain truncations of $M$ give rise to the same sheaf.

Lemma 6.8. Assume that $\mathcal{C} \subseteq \mathcal{K}$ and $\operatorname{dim}_{\mathbb{R}} \operatorname{pos}(\overline{\mathbf{C}})=r$. If $\boldsymbol{m} \in \mathbb{Z} \mathcal{K}$ and $M^{\prime}$ is the quotient module $M /\left.M\right|_{(\boldsymbol{m}+\mathbb{N} \mathbf{e})}$ then there exists $j>0$ such that $B^{j} M_{\boldsymbol{p}}^{\prime}=0$ for all 
$\boldsymbol{p} \in \mathbb{Z} \mathcal{K}$. Moreover, the module $M$ and its truncation $\left.M\right|_{(\boldsymbol{m}+\mathbb{N} \boldsymbol{e})}$ correspond to the same $\mathscr{O}_{X}$-module.

Proof. Fix $\boldsymbol{p} \in \mathbb{Z} \mathcal{K}$ and $f \in M_{\boldsymbol{p}}$. If $\boldsymbol{c} \in$ int $\mathcal{K}$, then Remark 3.7 (applied to the group $\mathbb{Z} \mathcal{K}$ ) shows that there is $k \in \mathbb{N}$ such that $\boldsymbol{p}+k \boldsymbol{c} \in \boldsymbol{m}+\mathcal{K}$. Since $\mathcal{C} \subseteq \mathcal{K}$ and $\operatorname{dim}_{\mathbb{R}} \operatorname{pos}(\overline{\mathbf{C}})=r$, there exists $\boldsymbol{q} \in \mathcal{K}$ for which $\boldsymbol{p}+k \boldsymbol{c}+\boldsymbol{q} \in \boldsymbol{m}+\mathbb{N} \mathcal{C}$. Now, Lemma 2.4 implies that $B \subseteq \sqrt{\left\langle S_{k \boldsymbol{c}+\boldsymbol{q}}\right\rangle}$ which means that there is $j>0$ such that $B^{j} \subseteq\left\langle S_{k \boldsymbol{c}+\boldsymbol{q}}\right\rangle$. Hence, we have $\left.B^{j} f \in M\right|_{(\boldsymbol{m}+\mathbb{N} \boldsymbol{e})}$. Because $M$ is finitely generated, there is one $j$ that works for all $f \in M_{\boldsymbol{p}}$ and all $\boldsymbol{p} \in \mathcal{K}$ which establishes the first part.

For the second part, consider the short exact sequence

$$
\left.0 \longrightarrow M\right|_{(\boldsymbol{m}+\mathbb{N} \mathbf{e})} \longrightarrow M \longrightarrow M^{\prime} \longrightarrow 0 \text {. }
$$

Since the functor $F \mapsto \widetilde{F}$ is exact, it suffices to show that $\widetilde{M^{\prime}}=0$. By Proposition 6.1] this is equivalent to the first part of the lemma.

Theorem 6.9. Assume that $\mathfrak{C} \subseteq \mathcal{K}$ and $\operatorname{dim}_{\mathbb{R}} \operatorname{pos}(\overline{\mathbf{C}})=r$. If $\boldsymbol{m} \in \mathbb{Z} \mathcal{K}$ and the sheaf $\mathscr{F}$ is $\boldsymbol{m}$-regular then the natural map

$$
H^{0}(X, \mathscr{F}(\boldsymbol{p})) \otimes H^{0}\left(X, \mathscr{O}_{X}(\boldsymbol{q})\right) \rightarrow H^{0}(X, \mathscr{F}(\boldsymbol{p}+\boldsymbol{q}))
$$

is surjective for all $\boldsymbol{p} \in \boldsymbol{m}+\mathbb{N} \boldsymbol{C}$ and all $\boldsymbol{q} \in \mathbb{N} \boldsymbol{C}$. In particular, the sheaf $\mathscr{F}(\boldsymbol{p})$ is generated by its global sections.

Proof. Consider the $G$-graded $S$-module

$$
M:=\left.\left(\bigoplus_{\boldsymbol{p} \in G} H^{0}(X, \mathscr{F}(\boldsymbol{p}))\right)\right|_{(\boldsymbol{m}+\mathbb{N} \mathcal{e})} .
$$

Because $\mathscr{F}$ is the sheaf associated with the $S$-module $\bigoplus_{\boldsymbol{p} \in \mathcal{K}} H^{0}(X, \mathscr{F}(\boldsymbol{p}))$, Lemma 6.8 guarantees that $\widetilde{M}=\mathscr{F}$. Since $\mathscr{F}$ is $\boldsymbol{m}$-regular, Proposition 6.4 shows that $M$ is $\boldsymbol{m}$-regular from level 2. Combining the definition of $M$ with the exact sequence (6.3.1), we have $H_{B}^{i}(M)_{\boldsymbol{p}}=0$ for all $i=0,1$ and all $\boldsymbol{p} \in \boldsymbol{m}+\mathbb{N} \mathcal{C}$. Hence, $M$ is $\boldsymbol{m}$-regular which implies that $M$ is also $\boldsymbol{p}$-regular for all $\boldsymbol{p} \in \boldsymbol{m}+\mathbb{N} \mathcal{C}$. If $\boldsymbol{p} \in \boldsymbol{m}+\mathbb{N} \boldsymbol{C}$, then Theorem 5.4 shows that $\left.M\right|_{p}=\left.M\right|_{(\boldsymbol{p}+\mathbb{N} \mathcal{C})}$. In other words, $M_{\boldsymbol{p}} \cdot S_{\boldsymbol{q}}=M_{\boldsymbol{p}+\boldsymbol{q}}$ for all $\boldsymbol{q} \in \mathbb{N} \mathcal{C}$ and this is equivalent to (6.9.3) being surjective. Furthermore, Lemma 6.8 also guarantees that $\mathscr{F}$ is the sheaf associated to $\left.M\right|_{(\boldsymbol{p}+\mathbb{N} \mathcal{C})}$. Since $\left.M\right|_{p}=\left.M\right|_{(\boldsymbol{p}+\mathbb{N} \mathcal{C})}$, this establishes the last part of the theorem.

Proof of Theorem 1.4. Part 1 follows from Remark 6.3. In the introduction, $\mathcal{C}$ is the minimal generating set for $\mathcal{K}$. Therefore, Theorem 6.9 proves Parts 2 and 3.

When $\mathscr{F}=\mathscr{O}_{X}$, Theorem 6.9 has a classical geometric interpretation. Specifically, if $\boldsymbol{p} \in \operatorname{reg}\left(\mathscr{O}_{X}\right)$ and $\mathscr{O}_{X}(\boldsymbol{p})$ is an ample line bundle then Theorem 6.9 implies that the natural map $H^{0}\left(X, \mathscr{O}_{X}(\boldsymbol{p})\right) \otimes H^{0}\left(X, \mathscr{O}_{X}(j \boldsymbol{p})\right) \longrightarrow H^{0}\left(X, \mathscr{O}_{X}((j+1) \boldsymbol{p})\right)$ is surjective for all $j \geq 0$. It follows (see Exercise II.5.14 in $[\mathrm{Har})$ ) that $\mathscr{O}_{X}(\boldsymbol{p})$ embeds $X$ into $\mathbb{P}^{N}$ as a projectively normal variety where $N:=\operatorname{dim}_{\mathrm{k}} H^{0}\left(X, \mathscr{O}_{X}(\boldsymbol{m})\right)$

It is an interesting open problem to describe the set of ample line bundles on a $d$-dimensional toric variety $X$ which give a projectively normal embedding. When $\mathscr{O}_{X}(\boldsymbol{m})$ is an ample line bundle, [BGT] show that the complete linear series of the line bundle $\mathscr{O}_{X}((d-1) \boldsymbol{m})$ produces a projectively normal embedding of $X$. Moreover, this bound is sharp on some simplicial toric varieties. For smooth toric varieties, the 
question of whether the map $H^{0}\left(X, \mathscr{O}_{X}(\boldsymbol{p})\right) \otimes H^{0}\left(X, \mathscr{O}_{X}(\boldsymbol{q})\right) \longrightarrow H^{0}\left(X, \mathscr{O}_{X}(\boldsymbol{p}+\boldsymbol{q})\right)$ is surjective for $\mathscr{O}_{X}(\boldsymbol{p})$ an ample line bundle and $\mathscr{O}_{X}(\boldsymbol{q})$ a nef line bundle appears in Oda. This would imply that every ample line bundle induced a projectively normal embedding.

These questions motivate the study of when $\mathbf{0} \in \operatorname{reg}\left(\mathscr{O}_{X}\right)$.

Proposition 6.10. If $X$ is a finite product of projective spaces and $\mathfrak{C}$ is the set of unique minimal generators of $\mathcal{K}$, then the structure sheaf $\mathscr{O}_{X}$ is $\mathbf{0}$-regular.

Proof. We proceed by induction on the number of factors in $X$. When there is only one copy of projective space, Example 6.5 establishes the proposition. For the induction step, we prove the following stronger claim: Let $X$ be a smooth projective toric variety such that $\mathscr{O}_{X}$ is $\mathbf{0}$-regular with respect to $\left\{\boldsymbol{c}_{1}, \ldots, \boldsymbol{c}_{\ell}\right\}$. If $Z=X \times \mathbb{P}^{h}$ then $\mathscr{O}_{Z}$ is 0-regular with respect to $\left\{\left[\begin{array}{c}\boldsymbol{c}_{1} \\ \mathbf{0}\end{array}\right], \ldots,\left[\begin{array}{c}\boldsymbol{c}_{\ell} \\ \mathbf{0}\end{array}\right],\left[\begin{array}{l}\mathbf{0} \\ \mathbf{1}\end{array}\right]\right\}$.

To accomplish this, we first fix some notation. Let $\Delta$ and $\Sigma$ be the simplicial complexes arising from the fans of $X$ and $Z$ respectively. If the set $\left\{\boldsymbol{a}_{1}, \ldots, \boldsymbol{a}_{n}\right\}$ defines the $\mathbb{Z}^{r}$-grading on the homogeneous coordinate ring of $X$ then the homogeneous coordinate $\operatorname{ring} S=\mathbb{k}\left[x_{1}, \ldots, x_{n}, y_{0}, \ldots, y_{h}\right]$ has the $\mathbb{Z}^{r+1}$-grading induced by $\operatorname{deg}\left(x_{i}\right)=\left[\begin{array}{c}\boldsymbol{a}_{i} \\ \mathbf{0}\end{array}\right]$ and $\operatorname{deg}\left(y_{i}\right)=\left[\begin{array}{l}\mathbf{0} \\ \mathbf{1}\end{array}\right]$. For $\tau \subseteq[n]$ and $\sigma \subseteq\{0, \ldots, h\}$, we write $\mathcal{V}_{\tau \cup \sigma}$ for the subset of $\mathbb{Z}^{r+1}$ given by

$$
\left[\begin{array}{c}
\sum_{j \in \tau}-\boldsymbol{a}_{j} \\
-|\sigma|
\end{array}\right]+\mathbb{N}\left\{\left[\begin{array}{c}
\boldsymbol{a}_{j} \\
\mathbf{0}
\end{array}\right]: j \in \widehat{\tau}\right\}+\mathbb{N}\left\{\left[\begin{array}{c}
-\boldsymbol{a}_{j} \\
\mathbf{0}
\end{array}\right]: j \in \tau\right\}+\mathbb{N}\left\{\left[\begin{array}{l}
\mathbf{0} \\
\mathbf{1}
\end{array}\right]: \text { if }|\sigma| \neq h+1\right\}+\mathbb{N}\left\{\left[\begin{array}{c}
\mathbf{0} \\
-\mathbf{1}
\end{array}\right]: \text { if }|\sigma| \neq 0\right\} .
$$

We also set

$$
\mathcal{R}^{i}:=\left\{-\lambda_{0}\left[\begin{array}{l}
\mathbf{0} \\
1
\end{array}\right]-\lambda_{1}\left[\begin{array}{c}
\boldsymbol{c}_{1} \\
\mathbf{0}
\end{array}\right]-\cdots-\lambda_{\ell}\left[\begin{array}{c}
\boldsymbol{c}_{\ell} \\
\mathbf{0}
\end{array}\right]: \lambda_{0}, \ldots, \lambda_{\ell} \in \mathbb{N} \text { and } \lambda_{0}+\cdots+\lambda_{\ell}=i\right\} .
$$

Now, Corollary 3.4 implies that $\mathscr{O}_{Z}$ is $\mathbf{0}$-regular if $\mathcal{V}_{\tau \cup \sigma} \cap \mathcal{R}^{i}=\emptyset$ whenever $i>0$ and $\widetilde{H}^{i-1}\left(\Sigma_{\tau \cup \sigma}\right) \neq 0$. We consider four possible cases:

- If $\emptyset \neq \sigma \neq\{0, \ldots, h\}$, then every maximal simplex in $\Sigma_{\tau \cup \sigma}$ contains $\sigma$. Since the induced subcomplex associated to $\sigma$ is the standard simplex, it follows that $\Sigma_{\tau \cup \sigma}$ is contractible and $\widetilde{H}^{i-1}\left(\Sigma_{\tau \cup \sigma}\right)=0$.

- If $\sigma=\emptyset$ then $\Sigma_{\tau \cup \sigma}$ is a subcomplex of $\Delta$. Since $\mathscr{O}_{X}$ is $\mathbf{0}$-regular, we must have $\mathcal{V}_{\tau \cup \sigma} \cap \mathcal{R}^{i}=\emptyset$ whenever $i>0$ and $\widetilde{H}^{i-1}\left(\Sigma_{\tau \cup \sigma}\right) \neq 0$.

- If $\sigma=\{0, \ldots, h\}$ and $\tau \neq[n]$, then Alexander duality implies that $\widetilde{H}^{i-1}\left(\Sigma_{\tau \cup \sigma}\right)$ is isomorphic to $\widetilde{H}^{d+h-i-1}\left(\Sigma_{\widetilde{\tau \cup \sigma}}\right)$ which reduces us to the second case.

- If $\sigma=\{0, \ldots, h\}$ and $\tau=[n]$ then $\widetilde{H}^{i-1}\left(\Sigma_{\tau \cup \sigma}\right) \neq 0$ if and only if $i=d+h$. Since both $\mathscr{O}_{\mathbb{P} d}$ and $\mathscr{O}_{X}$ are $\mathbf{0}$-regular, we must have $\mathcal{V}_{\tau \cup \sigma} \cap \mathcal{R}^{d+d^{\prime}}=\emptyset$.

The following example demonstrates that even when $X$ is a smooth Fano toric variety the structure sheaf $\mathscr{O}_{X}$ may not be $\mathbf{0}$-regular.

Example 6.11. Let $X$ be a blowup of $\mathbb{P}^{2}$ at three points. Specifically, the lattice points $\mathcal{B}$ correspond to the columns of the matrix $\left[\begin{array}{cccccc}1 & 0 & -1 & -1 & 0 & 1 \\ 0 & 1 & 1 & 0 & -1 & -1\end{array}\right]$. The regular triangulation of $\mathcal{B}$ is encoded in the irrelevant ideal

$$
B=\left\langle x_{1} x_{2} x_{3} x_{4}, x_{2} x_{3} x_{4} x_{5}, x_{3} x_{4} x_{5} x_{6}, x_{1} x_{4} x_{5} x_{6}, x_{1} x_{2} x_{5} x_{6}, x_{1} x_{2} x_{3} x_{6}\right\rangle .
$$


We have $G=\mathbb{Z}^{4}$ and we may assume that $\mathcal{A}$ is given by the columns of the matrix

$$
\left[\begin{array}{cccccc}
1 & 0 & 0 & 0 & 1 & -1 \\
0 & 1 & -1 & 1 & 0 & 0 \\
0 & 0 & 1 & -1 & 1 & 0 \\
0 & 0 & 0 & 1 & -1 & 1
\end{array}\right] .
$$

Let $\mathcal{C}$ be the unique minimal generators of the monoid $\mathcal{K}=\mathcal{K}^{\text {sat }}$ :

$$
\boldsymbol{C}=\left\{\left[\begin{array}{l}
1 \\
1 \\
0 \\
0
\end{array}\right],\left[\begin{array}{l}
0 \\
0 \\
1 \\
0
\end{array}\right],\left[\begin{array}{l}
1 \\
0 \\
1 \\
0
\end{array}\right],\left[\begin{array}{l}
0 \\
0 \\
0 \\
1
\end{array}\right],\left[\begin{array}{l}
0 \\
1 \\
0 \\
1
\end{array}\right]\right\} .
$$

It follows from Corollary 3.4 that

$$
H^{2}\left(X, \mathscr{O}_{X}\left(-\boldsymbol{c}_{3}-\boldsymbol{c}_{5}\right)\right)=H^{2}\left(X, \mathscr{O}_{X}\left(-\boldsymbol{a}_{1}-\boldsymbol{a}_{2}-\boldsymbol{a}_{3}-\boldsymbol{a}_{4}-\boldsymbol{a}_{5}-\boldsymbol{a}_{6}\right)\right)=\widetilde{H}^{1}(\Delta) \neq 0
$$

which implies $\mathbf{0} \notin \operatorname{reg}\left(\mathscr{O}_{X}\right)$.

The following remains an interesting open problem.

Problem 6.12. Let $\mathcal{C}$ be a set of minimal generators of $\mathcal{K}$. Give a combinatorial characterization of all toric varieties $X$ such that $\mathcal{K} \subseteq \operatorname{reg}\left(\mathscr{O}_{X}\right)$.

We end this section with an upper semi-continuity result. This is well-known when $X=\mathbb{P}^{d}$. We write $\operatorname{in}(I)$ for the initial ideal of an ideal $I \subseteq S$ with respect to some monomial order.

Proposition 6.13. Let $S$ be the homogeneous coordinate ring of a simplicial toric variety. If $I$ is an ideal in $S$, then $\operatorname{reg}(S / \operatorname{in}(I)) \subseteq \operatorname{reg}(S / I)$. Moreover, if I is B-saturated and $J=\left(\operatorname{in}(I): B^{\infty}\right)$ then $\operatorname{reg}(S / J) \subseteq \operatorname{reg}(S / I)$.

Proof. Fix $j>0$. If $\boldsymbol{x}^{\boldsymbol{u}} f$ belongs to $I$ for all $\boldsymbol{x}^{\boldsymbol{u}} \in B^{j}$, then $\boldsymbol{x}^{\boldsymbol{u}}$ in $(f)$ belongs to $\operatorname{in}(I)$. Hence, $f \in\left(I: B^{\infty}\right)$ implies that $\operatorname{in}(f) \in\left(\operatorname{in}(I): B^{\infty}\right)$. We conclude that $\operatorname{dim}_{\mathbb{k}} H_{B}^{0}(S / I)_{\boldsymbol{p}} \leq \operatorname{dim}_{\mathbb{k}} H_{B}^{0}(S / \operatorname{in}(I))_{\boldsymbol{p}}$ for all $\boldsymbol{p} \in G$. Theorem 15.17 in Eis gives a flat family over $\mathbb{A}^{1}$ whose general fiber is $S / I$ and whose special fiber is $S /(\operatorname{in}(I))$. Since $H_{B}^{i+1}(F) \cong \bigoplus_{\boldsymbol{p} \in G} H^{i}(X, \mathscr{F}(\boldsymbol{p}))$ for all $i \geq 1$, Theorem 12.8 in [Har] yields $\operatorname{dim}_{\mathbb{k}} H_{B}^{i}(S / I)_{\boldsymbol{p}} \leq \operatorname{dim}_{\mathbb{k}} H_{B}^{i}(S / \operatorname{in}(I))_{\boldsymbol{p}}$ for all $i>1$. If $\mathscr{I}$ denotes the sheaf of ideals corresponding to $I$, then the exact sequence (6.3.1) gives

$$
\operatorname{dim}_{\mathbb{k}} H_{B}^{0}(S / I)_{\boldsymbol{p}}+\operatorname{dim}_{\mathbb{k}} H^{0}\left(X,\left(\mathscr{O}_{X} / \mathscr{I}\right)(\boldsymbol{p})\right)=\operatorname{dim}_{\mathbb{k}}(S / I)_{\boldsymbol{p}}+\operatorname{dim}_{\mathbb{k}} H_{B}^{1}(S / I)_{\boldsymbol{p}} .
$$

Since the terms on left-hand side do not decrease and $\operatorname{dim}_{\mathbb{k}}(S / I)_{\boldsymbol{p}}$ is constant when passing to an initial ideal, we see that $\operatorname{dim}_{\mathbb{k}} H_{B}^{1}(S / I)_{\boldsymbol{p}}$ also does not decrease when passing to an initial ideal. Thus, we conclude that $H_{B}^{i}(S / \operatorname{in}(I))_{\boldsymbol{p}}=0$ implies $H_{B}^{i}(S / I)_{\boldsymbol{p}}=0$

and the first assertion follows. Since $H_{B}^{0}(S / J)=0$ and $H_{B}^{i}(S / J)=H_{B}^{i}(S / \operatorname{in}(I))$ for $i>0$, this also establishes the second assertion.

\section{Syzygies and Chain Complexes}

The final section of the paper examines the relationship between the regularity of $M$ and the syzygies of $M$. We give a combinatorial formula, involving only the degrees of the syzygies and the regularity of $S$, for a subset of $\operatorname{reg}(M)$. When $S$ is the homogeneous coordinate ring of weighted projective space, this subset actually equals $\operatorname{reg}(M)$. In other words, we recover the characterization of regularity in terms of Betti numbers in 
this case; see Theorem 5.5 in Ben. We extend this formula to coherent $\mathscr{O}_{X^{-}}$-modules. Changing directions, we then describe chain complexes associated to certain elements of $\operatorname{reg}(M)$. At the level of $\mathscr{O}_{X}$-modules, we obtain a locally free resolution of $\mathscr{F}$ from specific elements in $\operatorname{reg}(\mathscr{F})$.

If $\mathbf{E}$ is a free resolution of the module $M$, then there is a spectral sequence that computes the local cohomology of $M$ from $\mathbf{E}$, namely $E_{2}^{i, j}:=H^{i}\left(H_{B}^{j}(\mathbf{E})\right) \Longrightarrow H_{B}^{i+j}(M)$. When $S$ is the homogeneous coordinate ring of weighted projective space, this spectral sequence degenerates because $H_{B}^{i}(S)$ vanishes unless $i=d+1$; see Remark 3.3. As a consequence, there is a simple characterization of the regularity of $M$ in terms of the degrees of the syzygies. In contrast, Remark 3.3 also shows that nonvanishing local cohomology of $S$ is not typically concentrated in a single cohomological degree. Hence, the spectral sequence does not degenerate and one cannot expect as simple a relationship between regularity and syzygies in the general situation. Despite this, the syzygies of $M$ do provide a method for approximating the regularity of $M$ which captures the description in the special case. Moreover, this technique also works on a larger class of chain complexes associated to $M$.

We start by describing how regularity behaves in short exact sequences.

Lemma 7.1. If $0 \longrightarrow M^{\prime} \longrightarrow M \longrightarrow M^{\prime \prime} \longrightarrow 0$ is a short exact sequence of finitely generated $G$-graded $S$-modules, then we have the following:

1. $\operatorname{reg}^{i}\left(M^{\prime}\right) \cap \operatorname{reg}^{i}\left(M^{\prime \prime}\right) \subseteq \operatorname{reg}^{i}(M)$,

2. $\left(\bigcup_{1 \leq j \leq \ell}\left(-\boldsymbol{c}_{j}+\operatorname{reg}^{i+1}\left(M^{\prime}\right)\right)\right) \cap \operatorname{reg}^{i}(M) \subseteq \operatorname{reg}^{i}\left(M^{\prime \prime}\right)$ and

3. $\operatorname{reg}^{i}(M) \cap\left(\bigcap_{1 \leq j \leq \ell}\left(\boldsymbol{c}_{j}+\operatorname{reg}^{i-1}\left(M^{\prime \prime}\right)\right)\right) \subseteq \operatorname{reg}^{i}\left(M^{\prime}\right)$.

Proof. The associated long exact sequence in cohomology contains the exact sequence

$$
H_{B}^{i-1}\left(M^{\prime \prime}\right)_{\boldsymbol{p}} \longrightarrow H_{B}^{i}\left(M^{\prime}\right)_{\boldsymbol{p}} \longrightarrow H_{B}^{i}(M)_{\boldsymbol{p}} \longrightarrow H_{B}^{i}\left(M^{\prime \prime}\right)_{\boldsymbol{p}} \longrightarrow H_{B}^{i+1}\left(M^{\prime}\right)_{\boldsymbol{p}}
$$

Suppose that both $M^{\prime}$ and $M^{\prime \prime}$ are $\boldsymbol{m}$-regular from level $k$. This means that the second and fourth modules in (7.1.1) vanish for all $i \geq k$ and all $\boldsymbol{p} \in \boldsymbol{m}+\mathbb{N} \mathfrak{C}[1-i]$. Hence, the third module in (7.1.1) also vanishes for all $i \geq k$ and all $\boldsymbol{p} \in \boldsymbol{m}+\mathbb{N} \mathfrak{C}[1-i]$ which implies that $M$ is $\boldsymbol{m}$-regular from level $k$.

Similarly, if $M$ is $\boldsymbol{m}$-regular from level $k$ and $M^{\prime}$ is $\left(\boldsymbol{m}+\boldsymbol{c}_{j}\right)$-regular from level $(k+1)$ for some $1 \leq j \leq \ell$, then the third and fifth modules in (7.1.1) vanish for all $i \geq k$ and all $\boldsymbol{p} \in \boldsymbol{m}+\mathbb{N} \mathcal{C}[1-i]$. It follows that the fourth module in (7.1.1) also vanishes under the same conditions. Thus, $M^{\prime \prime}$ is $\boldsymbol{m}$-regular from level $k$.

Finally, if $M$ is $\boldsymbol{m}$-regular from level $k$ and $M^{\prime \prime}$ is $\left(\boldsymbol{m}-\boldsymbol{c}_{j}\right)$-regular from level $(k-1)$ for every $1 \leq j \leq \ell$, then the first and third modules in (7.1.1) vanish for all $i \geq 0$ and all $\boldsymbol{p} \in \boldsymbol{m}+\mathbb{N} \boldsymbol{e}[1-i]$. Hence, the second module in (7.1.1) vanishes under the same conditions and therefore $\boldsymbol{m} \in \operatorname{reg}^{k}\left(M^{\prime}\right)$.

The difference between the union in Part 2 and the intersection in Part 3 introduces an asymmetry in working with short exact sequences. In many cases, this prevents one from giving a simple characterization of $\boldsymbol{m}$-regularity in terms of minimal free resolutions. However, when $\mathcal{C}$ consists of a single element, as in the standard graded case, this obstruction is not present. In this case, we have the more symmetric: $\left(-\boldsymbol{c}_{1}+\right.$ $\left.\operatorname{reg}^{i+1}\left(M^{\prime}\right)\right) \cap \operatorname{reg}^{i}(M) \subseteq \operatorname{reg}^{i}(M)$ and $\left(\boldsymbol{c}_{1}+\operatorname{reg}^{i-1}\left(M^{\prime \prime}\right)\right) \cap \operatorname{reg}^{i}(M) \subseteq \operatorname{reg}^{i}\left(M^{\prime}\right)$. 
The approach used in the proof of Lemma 7.1 also leads to an analogous result for a short exact sequence of coherent $\mathscr{O}_{X}$-modules. However, there is a notable change in Part 3. Specifically, we must assume that $i>1$ because the hypothesis that $\mathscr{F}$ is $\boldsymbol{m}$-regular does not place any conditions on $H^{0}(X, \mathscr{F}(\boldsymbol{p}))$.

The next theorem provides a method for estimating the regularity of a module $M$ from certain chain complexes associated to $M$. We say a chain complex of $S$-modules has $B$-torsion homology if every homology module is a $B$-torsion module.

Theorem 7.2. Let $\mathbf{E}:=\cdots \longrightarrow E_{3} \stackrel{\partial_{3}}{\longrightarrow} E_{2} \stackrel{\partial_{2}}{\longrightarrow} E_{1} \stackrel{\partial_{1}}{\longrightarrow} E_{0} \longrightarrow 0$ be a chain complex of finitely generated $G$-graded $S$-modules with $B$-torsion homology. If $\partial_{0}: E_{0} \longrightarrow M$ is a surjective map then we have

$$
\bigcup_{\phi:[d+1] \rightarrow[\ell]}\left(\bigcap_{0 \leq i \leq d+1}\left(-\boldsymbol{c}_{\phi(1)}-\cdots-\boldsymbol{c}_{\phi(i)}+\operatorname{reg}^{i}\left(E_{i}\right)\right)\right) \subseteq \operatorname{reg}(M),
$$

where the union is over all functions $\phi:[d+1] \longrightarrow[\ell]$.

Proof. Fix a function $\phi:[d+1] \longrightarrow[\ell]$. We claim that for all $k \geq 0$

$$
\bigcap_{k \leq i \leq d+1}\left(-\boldsymbol{c}_{\phi(k+1)}-\cdots-\boldsymbol{c}_{\phi(i)}+\operatorname{reg}^{i}\left(E_{i}\right)\right) \subseteq \operatorname{reg}^{k}\left(\operatorname{Im} \partial_{k}\right) .
$$

Since $\operatorname{Im} \partial_{0}=M$, this will prove the theorem. We establish the claim by using a descending induction on $k$. Since $H_{B}^{j}\left(\operatorname{Im} \partial_{k}\right)$ vanishes for all $j>d+1$, we have $\operatorname{reg}^{k}\left(\operatorname{Im} \partial_{k}\right)=G$ for $k>d+1$ and the claim holds. Suppose $k \leq d+1$ and consider the exact sequence $0 \longrightarrow H_{k}(\mathbf{E}) \longrightarrow E_{k} / \operatorname{Im} \partial_{k+1} \longrightarrow \operatorname{Im} \partial_{k} \longrightarrow 0$. Because $H_{k}(\mathbf{E})$ is a $B$-torsion module, the associated long exact sequence in cohomology implies that $H_{B}^{j}\left(E_{k} / \operatorname{Im} \partial_{k+1}\right)=H_{B}^{j}\left(\operatorname{Im} \partial_{k}\right)$ for all $j>0$ and that $H_{B}^{0}\left(E_{k} / \operatorname{Im} \partial_{k+1}\right)$ surjects onto $H_{B}^{0}\left(\operatorname{Im} \partial_{k}\right)$. It follows that $\operatorname{reg}^{k}\left(E_{k} / \operatorname{Im} \partial_{k+1}\right) \subseteq \operatorname{reg}^{k}\left(\operatorname{Im} \partial_{k}\right)$ for all $k \geq 0$. Applying Lemma 7.1 to the short exact sequence $0 \longrightarrow \operatorname{Im} \partial_{k+1} \longrightarrow E_{k} \longrightarrow E_{k} / \operatorname{Im} \partial_{k+1} \longrightarrow 0$ gives $\operatorname{reg}^{k+1}\left(\operatorname{Im} \partial_{k+1}\right)[-1] \cap \operatorname{reg}^{k}\left(E_{k}\right) \subseteq \operatorname{reg}^{k}\left(E_{k} / \operatorname{Im} \partial_{k+1}\right)$. Since the induction hypothesis implies that $\bigcap_{k+1 \leq i \leq d+1}\left(-\boldsymbol{c}_{\phi(k+2)}-\cdots-\boldsymbol{c}_{\phi(i)}+\operatorname{reg}^{i}\left(E_{i}\right)\right) \subseteq \operatorname{reg}^{k+1}\left(\operatorname{Im} \partial_{k+1}\right)$, we have $\bigcap_{k+1 \leq i \leq d+1}\left(-\boldsymbol{c}_{\phi(k+1)}-\cdots-\boldsymbol{c}_{\phi(i)}+\operatorname{reg}^{i}\left(E_{i}\right)\right) \subseteq \operatorname{reg}^{k+1}\left(\operatorname{Im} \partial_{k+1}\right)[-1]$. Combining these three inclusions, we obtain $\bigcap_{k \leq i \leq d+1}\left(-\boldsymbol{c}_{\phi(k+1)}-\cdots-\boldsymbol{c}_{\phi(i)}+\operatorname{reg}^{i}\left(E_{i}\right)\right) \subseteq \operatorname{reg}^{k}\left(\operatorname{Im} \partial_{k}\right)$.

Since the proof of Theorem [7.2 only used Part 2 of Lemma 7.1, the same approach leads to a formula for estimating the regularity of a sheaf $\mathscr{F}$ from a resolution. We leave the details to the interested reader. Because it is frequently used in applications, we explicitly state the following special case of Theorem [7.2.

Corollary 7.3. If $0 \longrightarrow E_{s} \longrightarrow \cdots \longrightarrow E_{2} \longrightarrow E_{1} \longrightarrow E_{0}$ is a free resolution of the module $M$ with $E_{i}=\bigoplus_{1 \leq j \leq h_{i}} S\left(-\boldsymbol{q}_{i, j}\right)$ for some $\boldsymbol{q}_{i, j} \in G$, then we have

$$
\bigcup_{\phi:[d+1] \rightarrow[\ell]}\left(\bigcap_{\substack{0 \leq i \leq \min \{d+1, s\} \\ 1 \leq j \leq h_{i}}}\left(\boldsymbol{q}_{i, j}-\boldsymbol{c}_{\phi(1)}-\cdots-\boldsymbol{c}_{\phi(i)}+\operatorname{reg}^{i}(S)\right)\right) \subseteq \operatorname{reg}(M) .
$$

Proof. Since $\operatorname{reg}^{i}\left(E_{i}\right)=\bigcap_{1 \leq j \leq h_{i}}\left(\boldsymbol{q}_{i, j}+\operatorname{reg}^{i}(S)\right)$, (7.3.2) follows from Theorem 7.2

The next three examples illustrate this corollary. 
Example 7.4. Suppose $S$ is the homogeneous coordinate ring of weighted projective space; see Example 2.1. Let $\mathcal{C}=\{\mathbf{1}\}$ and let $0 \longrightarrow E_{s} \longrightarrow \cdots \longrightarrow E_{2} \longrightarrow E_{1} \longrightarrow E_{0}$ be the minimal free resolution of the module $M$ where $E_{i}=\bigoplus_{1 \leq j \leq h_{i}} S\left(-\boldsymbol{q}_{i, j}\right)$ for some $\boldsymbol{q}_{i, j} \in \mathbb{Z}$. Hilbert's syzygy theorem implies that $s \leq n=d+1$. Hence, Example 4.2 and (17.3.2) imply that $\max _{i, j}\left\{\boldsymbol{q}_{i, j}-i+n-\boldsymbol{a}_{1}-\cdots-\boldsymbol{a}_{n}\right\} \in \operatorname{reg}(M)$. In particular, if $S$ has the standard grading, then $M$ is $\left(\max _{i, j}\left\{\boldsymbol{q}_{i, j}-i\right\}\right)$-regular.

Example 7.5. Let $S$ be the homogeneous coordinate ring of $\mathbb{P}^{1} \times \mathbb{P}^{1} \times \mathbb{P}^{1}$. This means that $\mathcal{B}$ corresponds to the columns of the matrix

$$
\left[\begin{array}{cccccc}
1 & -1 & 0 & 0 & 0 & 0 \\
0 & 0 & 1 & -1 & 0 & 0 \\
0 & 0 & 0 & 0 & 1 & -1
\end{array}\right] \text {. }
$$

and the irrelevant ideal is $B=\left\langle x_{1}, x_{2}\right\rangle \cap\left\langle x_{3}, x_{4}\right\rangle \cap\left\langle x_{5}, x_{6}\right\rangle$. Hence, $G=\mathbb{Z}^{3}$ and we may assume that $\mathcal{A}$ is given by the columns of the matrix

$$
\left[\begin{array}{llllll}
1 & 1 & 0 & 0 & 0 & 0 \\
0 & 0 & 1 & 1 & 0 & 0 \\
0 & 0 & 0 & 0 & 1 & 1
\end{array}\right] .
$$

The polynomial ring $S=\mathbb{k}\left[x_{1}, \ldots, x_{6}\right]$ has the corresponding $\mathbb{Z}^{3}$-grading and $\mathcal{K}=$ $\mathcal{K}^{\text {sat }}=\mathbb{N}^{3}$. If $\mathcal{C}=\left\{\boldsymbol{c}_{1}, \boldsymbol{c}_{2}, \boldsymbol{c}_{3}\right\} \subset \mathcal{K}$ where $\boldsymbol{c}_{i}$ is the $i$ th standard basis vector in $\mathbb{Z}^{3}$, then Corollary 3.4) implies that $\operatorname{reg}(S)=\mathbb{N}^{3}$.

Consider the module $M=S / I$ where $I=\left\langle x_{1}-x_{2}, x_{3}-x_{4}, x_{5}-x_{6}\right\rangle$. Since $I$ is a reduced prime ideal of codimension 3 , the subvariety of $\mathbb{P}^{1} \times \mathbb{P}^{1} \times \mathbb{P}^{1}$ defined by $I$ is a single point. The minimal free resolution of $M$ has the form

$$
0 \longrightarrow S\left(-\boldsymbol{c}_{1}-\boldsymbol{c}_{2}-\boldsymbol{c}_{3}\right) \longrightarrow \bigoplus_{1 \leq i<j \leq 3} S\left(-\boldsymbol{c}_{i}-\boldsymbol{c}_{j}\right) \longrightarrow \bigoplus_{1 \leq i \leq 3} S\left(-\boldsymbol{c}_{i}\right) \longrightarrow S .
$$

A calculation using Corollary [7.3] shows that $\bigcup_{1 \leq i<j \leq 3}\left(\boldsymbol{c}_{i}+\boldsymbol{c}_{j}+\mathbb{N}^{3}\right) \subseteq \operatorname{reg}(M)$. Note that this is not the entire regularity of $M$. Proposition 6.7 show that $\operatorname{reg}(M)=\mathbb{N}^{3}$.

Example 7.6. As in Example 7.5. we assume that $S$ is the homogeneous coordinate ring of the toric variety $\mathbb{P}^{1} \times \mathbb{P}^{1} \times \mathbb{P}^{1}$ and we choose $\mathcal{C}$ to be the set of standard basis vectors in $\mathbb{Z}^{3}$. Consider $M=S / I$ where $I=\left\langle x_{1}-x_{2}, x_{3}-x_{4}, x_{5}-x_{6}\right\rangle \cap\left\langle x_{1}-2 x_{2}, x_{3}-2 x_{4}, x_{5}-2 x_{6}\right\rangle$. The $B$-saturated ideal $I$ corresponds to two distinct points on $\mathbb{P}^{1} \times \mathbb{P}^{1} \times \mathbb{P}^{1}$. The minimal free resolution of $M$ has the form

$$
0 \rightarrow \bigoplus_{\boldsymbol{q} \in \mathcal{D}_{5}} S(-\boldsymbol{q}) \rightarrow \bigoplus_{\boldsymbol{q} \in \mathcal{D}_{4}} S(-\boldsymbol{q}) \rightarrow \bigoplus_{\boldsymbol{q} \in \mathcal{D}_{3}} S(-\boldsymbol{q}) \rightarrow \bigoplus_{\boldsymbol{q} \in \mathcal{D}_{2}} S(-\boldsymbol{q}) \rightarrow \bigoplus_{\boldsymbol{q} \in \mathcal{D}_{1}} S(-\boldsymbol{q}) \rightarrow S
$$

where, for $1 \leq i \leq 5$, the set $\mathcal{D}_{i}$ is given by the column vectors of the matrix $D_{i}$ and

$$
\begin{aligned}
& D_{1}=\left[\begin{array}{lllllllll}
2 & 1 & 1 & 1 & 1 & 0 & 0 & 0 & 0 \\
0 & 1 & 1 & 0 & 0 & 2 & 1 & 1 \\
0 & 0 & 0 & 1 & 1 & 0 & 1 & 1 & 1 \\
0
\end{array}\right] \text {, }
\end{aligned}
$$

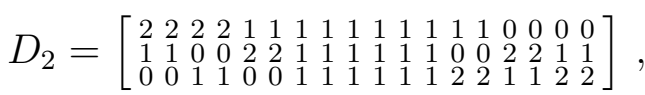

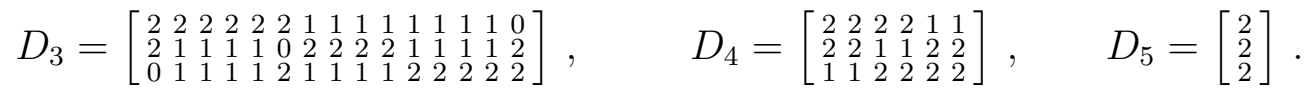

Observe that the length of the minimal free resolution is greater than $d+1$. Applying Corollary [7.3] we deduce that

$$
\left(\left[\begin{array}{l}
1 \\
2 \\
2
\end{array}\right]+\mathbb{N}^{3}\right) \cup\left(\left[\begin{array}{l}
2 \\
1 \\
2
\end{array}\right]+\mathbb{N}^{3}\right) \cup\left(\left[\begin{array}{l}
2 \\
2 \\
1
\end{array}\right]+\mathbb{N}^{3}\right) \subset \operatorname{reg}(M) .
$$


However, Proposition 6.7 shows that

$$
\operatorname{reg}(M)=\left(\left[\begin{array}{l}
1 \\
0 \\
0
\end{array}\right]+\mathbb{N}^{3}\right) \cup\left(\left[\begin{array}{l}
0 \\
1 \\
0
\end{array}\right]+\mathbb{N}^{3}\right) \cup\left(\left[\begin{array}{l}
0 \\
0 \\
1
\end{array}\right]+\mathbb{N}^{3}\right) .
$$

To describe a partial converse for Theorem 7.2, we concentrate on the situation arising from smooth toric varieties. Specifically, we assume that $\mathcal{K}=\mathcal{K}^{\text {sat }}$ and that $\mathcal{C}$ is a finite generating set for the monoid $\mathcal{K}$.

Lemma 7.7. Let $\mathbb{N} \mathcal{C}=\mathcal{K}=\mathcal{K}^{\text {sat }}$. If $M$ is $\boldsymbol{m}$-regular then $\left.M\right|_{(\boldsymbol{m}+\mathcal{K})}$ is also $\boldsymbol{m}$-regular .

Proof. Suppose that $M$ is $\boldsymbol{m}$-regular and let $M^{\prime}$ be the quotient module $M /\left.M\right|_{(\boldsymbol{m}+\boldsymbol{K})}$. Since $\mathbb{N} \mathcal{C}=\mathcal{K}=\mathcal{K}^{\text {sat }}$, Lemma 6.8 establishes that $M^{\prime}$ is a $B$-torsion module. Hence, the long exact sequence associated to (6.8.2) implies that $H_{B}^{i}\left(\left.M\right|_{(\boldsymbol{m}+\mathcal{K})}\right)=H_{B}^{i}(M)$ for all $i>1$ and $H_{B}^{1}\left(\left.M\right|_{(\boldsymbol{m}+\mathcal{K})}\right)_{\boldsymbol{p}}=H_{B}^{1}(M)_{\boldsymbol{p}}$ for all $\boldsymbol{p} \in \boldsymbol{m}+\mathcal{K}$. For $k>0$, we deduce that $M$ is $\boldsymbol{m}$-regular from level $k$ if and only if the submodule $\left.M\right|_{(\boldsymbol{m}+\mathcal{K})}$ is $\boldsymbol{m}$-regular from level $k$. The long exact sequence also shows that $H_{B}^{0}\left(\left.M\right|_{(\boldsymbol{m}+\mathcal{K})}\right)$ is a submodule of $H_{B}^{0}(M)$. Thus, if $H_{B}^{0}(M)_{\boldsymbol{p}}=0$ then $H_{B}^{0}\left(\left.M\right|_{(\boldsymbol{m}+\mathcal{K})}\right)_{\boldsymbol{p}}$ also vanishes. Therefore, $\left.M\right|_{(\boldsymbol{m}+\mathcal{K})}$ is $\boldsymbol{m}$-regular.

Theorem 7.8. Assume that $\mathbb{N} \mathcal{C}=\mathcal{K}=\mathcal{K}^{\text {sat }}$. If $\boldsymbol{c} \in \operatorname{reg}(S) \cap\left(\bigcap_{1 \leq j \leq \ell}\left(\boldsymbol{c}_{j}+\mathcal{K}\right)\right)$ and $\boldsymbol{m} \in \operatorname{reg}(M)$ then there exists

1. a chain complex $\cdots \longrightarrow E_{3} \stackrel{\partial_{3}}{\longrightarrow} E_{2} \stackrel{\partial_{2}}{\longrightarrow} E_{1} \stackrel{\partial_{1}}{\longrightarrow} E_{0}$ with B-torsion homology and $E_{i}=\bigoplus_{1 \leq j \leq h_{i}} S(-\boldsymbol{m}-i \boldsymbol{c})$, and

2. a surjective map $\partial_{0}:\left.E_{0} \longrightarrow M\right|_{(\boldsymbol{m}+\mathbb{N} \mathbf{e})}$.

Proof. We prove by induction on $k$ that there exists a chain complex with $B$-torsion homology

$$
0 \longrightarrow M_{k} \longrightarrow E_{k-1} \stackrel{\partial_{k-1}}{\longrightarrow} \cdots \stackrel{\partial_{1}}{\longrightarrow} E_{0} \stackrel{\partial_{0}}{\longrightarrow} M \stackrel{\partial_{-1}}{\longrightarrow} 0
$$

such that $E_{i}=\bigoplus_{1 \leq j \leq h_{i}} S(-\boldsymbol{m}-i \boldsymbol{c}), M_{k}=\operatorname{Ker} \partial_{k-1}$ and $\boldsymbol{m}+k \boldsymbol{c} \in \operatorname{reg}\left(M_{k}\right)$. Since $M=M_{0}$ and $M$ is $\boldsymbol{m}$-regular, the first step in the induction holds.

Suppose $k \geq 0$. Since $\mathbb{N} \mathcal{C}=\mathcal{K}=\mathcal{K}^{\text {sat }}$, Lemma 7.7 and Lemma 6.8 show that $\operatorname{reg}\left(M_{k}\right) \subseteq \operatorname{reg}\left(\left.M_{k}\right|_{(\boldsymbol{m}+k \boldsymbol{c}+\mathbb{N} \boldsymbol{e})}\right)$ and that $M_{k} /\left.M_{k}\right|_{(\boldsymbol{m}+k \boldsymbol{c}+\mathbb{N} \boldsymbol{e})}$ is a $B$-torsion module. The induction hypothesis states that $M_{k}$ is $(\boldsymbol{m}+k \boldsymbol{c})$-regular and Theorem 5.4 implies that $\left.M_{k}\right|_{(\boldsymbol{m}+k \boldsymbol{c}+\mathbb{N} \boldsymbol{e})}$ is generated in degree $\boldsymbol{m}+k \boldsymbol{c}$. It follows that there exists a surjective map $\partial_{k}:\left.E_{k} \longrightarrow M_{k}\right|_{(\boldsymbol{m}+k \boldsymbol{c}+\mathbb{N} \boldsymbol{e})}$ where $E_{k}:=\bigoplus_{1 \leq j \leq b_{k}} S(-\boldsymbol{m}-k \boldsymbol{c})$. Setting $M_{k+1}:=\operatorname{Ker} \partial_{k}$, we obtain the short exact sequence

$$
\left.0 \longrightarrow M_{k+1} \longrightarrow E_{k} \longrightarrow M_{k}\right|_{(\boldsymbol{m}+k \boldsymbol{c}+\mathbb{N} \mathbf{e})} \longrightarrow 0
$$

Combining (7.8.3) and (7.8.4) gives the chain complex with $B$-torsion homology

$$
0 \longrightarrow M_{k+1} \longrightarrow E_{k} \stackrel{\partial_{k}}{\longrightarrow} \cdots \stackrel{\partial_{1}}{\longrightarrow} E_{0} \stackrel{\partial_{0}}{\longrightarrow} M \stackrel{\partial_{-1}}{\longrightarrow} 0
$$

where $E_{i}=\bigoplus_{1 \leq j \leq h_{i}} S(-\boldsymbol{m}-i \boldsymbol{c})$ and $M_{k+1}=\operatorname{Ker} \partial_{k}$. It remains to show that $M_{k+1}$ is $(\boldsymbol{m}+(k+1) \boldsymbol{c})$-regular. Applying Lemma [7.1] to (7.8.4) yields

$$
(\boldsymbol{m}+k \boldsymbol{c}+\operatorname{reg}(S)) \cap \bigcap_{1 \leq i \leq \ell}\left(\boldsymbol{c}_{i}+\operatorname{reg}\left(M_{k}\right)\right) \subseteq \operatorname{reg}\left(M_{k+1}\right) .
$$


Our choice of $\boldsymbol{c}$ guarantees that $\boldsymbol{m}+(k+1) \boldsymbol{c}$ lies in both $\bigcap_{1 \leq i \leq \ell}\left(\boldsymbol{c}_{i}+\operatorname{reg}\left(M_{k}\right)\right)$ and $\boldsymbol{m}+k \boldsymbol{c}+\operatorname{reg}(S)$ which completes the proof.

Proof of Theorem 1.5. In the introduction, $X$ was smooth so $\mathcal{K}=\mathcal{K}^{\text {sat }}$. We also assumed that $\mathcal{C}$ was the set of minimal generators for $\mathcal{K}$. Hence, Theorem $\mathbf{7 . 2}$ establishes Part 1 and Theorem 7.8 proves Part 2.

This theorem leads to a "linear" resolution of $\mathscr{O}_{X}$-modules.

Corollary 7.9. Assume that $\mathbb{N} \mathcal{C}=\mathcal{K}=\mathcal{K}^{\text {sat }}$. If $\boldsymbol{c} \in \operatorname{reg}\left(\mathscr{O}_{X}\right) \cap\left(\bigcap_{1 \leq j \leq \ell}\left(\boldsymbol{c}_{j}+\mathcal{K}\right)\right)$ and $\boldsymbol{m} \in \operatorname{reg}(\mathscr{F})$ then there is an exact sequence

$$
\cdots \longrightarrow \mathscr{E}_{3} \longrightarrow \mathscr{E}_{2} \longrightarrow \mathscr{E}_{1} \longrightarrow \mathscr{E}_{0} \longrightarrow \mathscr{F} \longrightarrow 0
$$

where $\mathscr{E}_{i}=\bigoplus_{1 \leq j \leq h_{i}} \mathscr{O}_{X}(-\boldsymbol{m}-i \boldsymbol{c})$.

Proof. Consider the $G$-graded $S$-module

$$
M=\left.\left(\bigoplus_{\boldsymbol{p} \in G} H^{0}(X, \mathscr{F}(\boldsymbol{p}))\right)\right|_{(\boldsymbol{m}+\mathcal{K})} .
$$

Lemma 6.8 implies that $\widetilde{M}=\mathscr{F}$ and Proposition 6.4 shows that $M$ is $\boldsymbol{m}$-regular. Theorem 7.8 produces a chain complex $\cdots \longrightarrow E_{3} \longrightarrow E_{2} \longrightarrow E_{1} \longrightarrow E_{0} \longrightarrow M \longrightarrow 0$ with $B$-torsion homology and $E_{i}=\bigoplus_{1 \leq j \leq h_{i}} S(-\boldsymbol{m}-i \boldsymbol{c})$. Applying the functor $F \mapsto \widetilde{F}$, we obtain a chain complex of the desired form. Moreover, Proposition 6.1 implies that the homology of this complex consists of the zero sheaf. Therefore, the chain complex is a locally free resolution of $\mathscr{F}$.

We illustrate Theorem 7.8 with the following examples. The first example shows that Theorem 7.8 is a converse to Theorem $[7.2$ in the standard graded case.

Example 7.10. Let $S$ have the standard grading, let $B=\left\langle x_{1}, \ldots, x_{n}\right\rangle$, and let $\mathbf{C}=\{\mathbf{1}\}$. Since $\operatorname{reg}(S)=\mathbb{N}$, we have $\boldsymbol{c}=\mathbf{1} \in \operatorname{reg}(S) \cap(\mathbf{1}+\mathcal{K})$. Hence, if $\boldsymbol{m} \in \operatorname{reg}(M)$, then Theorem 7.8 implies that there is a chain complex $\mathbf{E}:=\cdots \longrightarrow E_{2} \longrightarrow E_{1} \longrightarrow E_{0}$ with $B$-torsion homology and $E_{i}=\bigoplus_{1 \leq j \leq h_{i}} S(-m-i)$ and there is a surjective map $\left.E_{0} \longrightarrow M\right|_{(\boldsymbol{m}+\mathbb{N})}$. Conversely, given a such chain complex, Example 7.4 shows that $\boldsymbol{m} \in \operatorname{reg}(M)$. In fact, Exercise 1.4.24 in $[\mathrm{BH}]$ proves that $\mathbf{E}$ must be a resolution.

Example 7.11. Let $M$ be the module in Example 7.5 and let

$$
\boldsymbol{c}:=\boldsymbol{c}_{1}+\boldsymbol{c}_{2}+\boldsymbol{c}_{3} \in \operatorname{reg}(S) \cap\left(\bigcap_{1 \leq j \leq 3}\left(\boldsymbol{c}_{j}+\mathbb{N} \mathcal{C}\right)\right)
$$

If $\boldsymbol{m}:=\mathbf{0} \in \operatorname{reg}(M)$, then Theorem 7.8 produces a chain complex with $B$-torsion homology of the form

$$
\cdots \longrightarrow \bigoplus_{i} S(-3 \boldsymbol{c}) \longrightarrow \bigoplus_{i} S(-2 \boldsymbol{c}) \longrightarrow \bigoplus_{1 \leq i \leq 7} S(-\boldsymbol{c}) \longrightarrow S
$$

However, applying Theorem $[7.2$ to this chain complex yields

$$
\bigcup_{\substack{p_{1}, p_{2}, p_{3} \in \mathbb{N} \\
p_{1}+p_{2}+p_{3}=8}}\left(\left[\begin{array}{c}
p_{1} \\
p_{2} \\
p_{3}
\end{array}\right]+\mathbb{N}^{3}\right) \subset \operatorname{reg}(M),
$$

and the smaller set does not contain $\mathbf{0 .}$ 


\section{REFERENCES}

[Ben] David Benson, Dickson invariants, regularity and computation in group cohomology, arXiv:math.GR/0303187.

[BGS] Louis J. Billera, Israel M. Gel'fand, and Bernd Sturmfels, Duality and minors of secondary polyhedra, Journal of Combinatorial Theory. Series B 57 (1993), no. 2, 258-268.

[BGT] Winfried Bruns, Joseph Gubeladze, and Ngô Viêt Trung, Normal polytopes, triangulations, and Koszul algebras, Journal für die Reine und Angewandte Mathematik 485 (1997), 123-160.

[BH] Winfried Bruns and Jürgen Herzog, Cohen-Macaulay rings, Cambridge Studies in Advanced Mathematics, vol. 39, Cambridge University Press, Cambridge, 1993.

[BK] Winfried Bruns and Robert Koch, Normaliz: Computing the normalization of affine semigroups, ftp://ftp.mathematik.uni-osnabrueck.de/pub/osm/kommalg/software/.

[BmS] David Bayer and Michael Stillman, A criterion for detecting m-regularity, Inventiones Mathematicae 87 (1987), no. 1, 1-11.

[BbS] Dave Bayer and Bernd Sturmfels, Cellular resolutions of monomial modules, Journal für die Reine und Angewandte Mathematik 502 (1998), 123-140.

[BrS] Markus P. Brodmann and Rodney Y. Sharp, Local cohomology: an algebraic introduction with geometric applications, Cambridge Studies in Advanced Mathematics, vol. 60, Cambridge University Press, Cambridge, 1998.

[Chi] Jaydeep V. Chipalkatti, A generalization of Castelnuovo regularity to Grassmann varieties, Manuscripta Mathematica 102 (2000), no. 4, 447-464.

[Co1] David A. Cox, Equations of Parametric Surfaces via Syzygies, arXiv:math.AG/0010107.

[Co2] David A. Cox, The homogeneous coordinate ring of a toric variety, Journal of Algebraic Geometry 4 (1995), no. 1, 17-50.

[Co3] - Recent developments in toric geometry, Algebraic geometry-Santa Cruz 1995, Proc. Sympos. Pure Math., vol. 62, American Mathematical Society, Providence, RI, 1997, pp. 389436.

[EG] David Eisenbud and Shiro Goto, Linear free resolutions and minimal multiplicity, Journal of Algebra 88 (1984), no. 1, 89-133.

[Eis] David Eisenbud, Commutative algebra with a view toward algebraic geometry, Graduate Texts in Mathematics, vol. 150, Springer-Verlag, New York, 1995.

[EL] Lawrence Ein and Robert Lazarsfeld, Syzygies and Koszul cohomology of smooth projective varieties of arbitrary dimension, Inventiones Mathematicae 111 (1993), no. 1, 51-67.

[EMS] David Eisenbud, Mircea Mustaţă, and Mike Stillman, Cohomology on toric varieties and local cohomology with monomial supports, Journal of Symbolic Computation 29 (2000), no. 4-5, 583-600.

[Ful] William Fulton, Introduction to toric varieties, Annals of Mathematics Studies 131, Princeton University Press, Princeton, NJ, 1993, The William H. Roever Lectures in Geometry.

[GLP] Laurent Gruson, Robert Lazarsfeld, and Christian Peskine, On a theorem of Castelnuovo, and the equations defining space curves, Inventiones Mathematicae 72 (1983), no. 3, 491-506.

[Got] Gerd Gotzmann, Eine Bedingung für die Flachheit und das Hilbertpolynom eines graduierten Ringes, Mathematische Zeitschrift 158 (1978), no. 1, 61-70.

[M2] Daniel R. Grayson and Michael E. Stillman, Macaulay 2, a software system for research in algebraic geometry, Available at http://www.math.uiuc.edu/Macaulay2.

[Har] Robin Hartshorne, Algebraic geometry, Graduate Texts in Mathematics, vol. 52, SpringerVerlag, New York, 1977.

[HmS] Mark Haiman and Bernd Sturmfels, Multigraded Hilbert schemes, arXiv:math. AG/0201271.

[HtS] Tamas Hausel and Bernd Sturmfels, Toric Hyperkahler Varieties, arXiv:math. AG/0203096.

[HV] Huy Tai Ha and Adam Van Tuyl, The regularity of points in multi-projective spaces, arXiv:math. AC/0211263.

[HW] J. William Hoffman and Hao Hao Wang, Castelnuovo-Mumford Regularity in Biprojective Spaces, arXiv:math.AG/0212033. 
[IK] Anthony Iarrobino and Vassil Kanev, Power sums, Gorenstein algebras, and determinantal loci, Lecture Notes in Mathematics, vol. 1721, Springer-Verlag, Berlin, 1999, Appendix C by Iarrobino and Steven L. Kleiman.

[Laz] Robert Lazarsfeld, Positivity in algebraic geometry, to appear.

[Mil] Ezra Miller, The Alexander duality functors and local duality with monomial support, Journal of Algebra 231 (2000), no. 1, 180-234.

[MS] Diane Maclagan and Gregory G. Smith, Uniform bounds on multigraded regularity, 2003.

[Mum] David Mumford, Lectures on curves on an algebraic surface, Princeton University Press, Princeton, N.J., 1966.

[Mun] James R. Munkres, Elements of algebraic topology, Addison-Wesley Publishing Company, Menlo Park, CA, 1984.

[Oda] Tadao Oda, Open problems on Minkowski sums of polytopes, 1997, preprint, Tôhoku University.

[PP] Giuseppe Pareschi and Mihnea Popa, Regularity on abelian varieties. I, Journal of the American Mathematical Society 16 (2003), no. 2, 285-302.

[Rob] Paul C. Roberts, Multiplicities and Chern classes in local algebra, Cambridge Tracts in Mathematics, vol. 133, Cambridge University Press, Cambridge, 1998.

[RWY] Victor Reiner, Volkmar Welker, and Kohji Yanagawa, Local cohomology of Stanley-Reisner rings with supports in general monomial ideals, Journal of Algebra 244 (2001), no. 2, 706-736.

[Zie] Günter M. Ziegler, Lectures on polytopes, Graduate Texts in Mathematics, vol. 152, SpringerVerlag, New York, 1995.

Department of Mathematics, Stanford University, Stanford, CA 94305, USA

E-mail address: maclagan@math.stanford.edu

Department of Mathematics, Barnard College, Columbia University, New York, NY 10027, USA

E-mail address: ggsmith@math.columbia.edu 